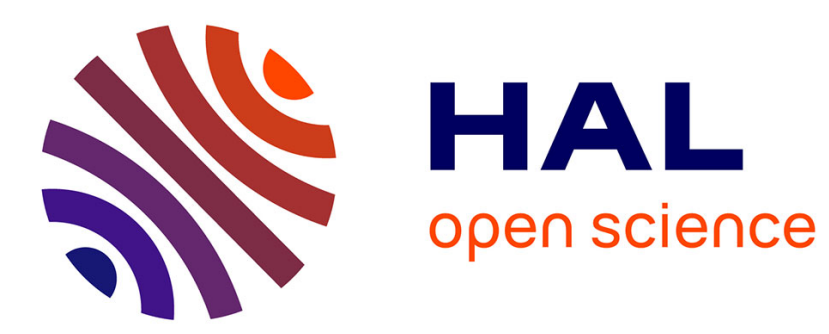

\title{
Chevron morphology in deformed semicrystalline polymers
}

\author{
Marina Krumova, Sven Henning, Goerg Hannes Michler
}

\section{To cite this version:}

Marina Krumova, Sven Henning, Goerg Hannes Michler. Chevron morphology in deformed semicrystalline polymers. Philosophical Magazine, 2006, 86 (12), pp.1689-1712. 10.1080/14786430500501671. hal-00513645

\section{HAL Id: hal-00513645 \\ https://hal.science/hal-00513645}

Submitted on 1 Sep 2010

HAL is a multi-disciplinary open access archive for the deposit and dissemination of scientific research documents, whether they are published or not. The documents may come from teaching and research institutions in France or abroad, or from public or private research centers.
L'archive ouverte pluridisciplinaire HAL, est destinée au dépôt et à la diffusion de documents scientifiques de niveau recherche, publiés ou non, émanant des établissements d'enseignement et de recherche français ou étrangers, des laboratoires publics ou privés. 


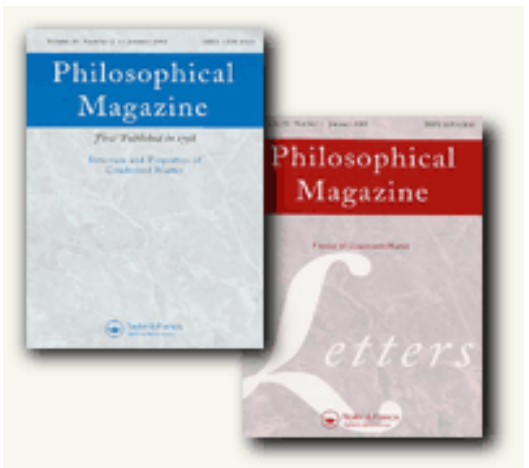

\section{Chevron morphology in deformed semicrystalline polymers}

\begin{tabular}{|r|l|}
\hline Journal: & Philosophical Magazine \& Philosophical Magazine Letters \\
\hline Manuscript ID: & TPHM-05-Mar-0068.R2 \\
\hline Journal Selection: & Philosophical Magazine \\
\hline Author: & $25-$ Subt-2005 \\
\hline Complete List of Authors: & $\begin{array}{l}\text { Krumova, Marina; University Halle-Wittenberg, Physics } \\
\text { Henning, Sven; University Halle-Wittenberg, Materials Science } \\
\text { Michler, Goerg Hannes; University Halle-Wittenberg, Materials } \\
\text { Science }\end{array}$ \\
\hline Keywords: & TEM, deformation, morphology, semicrystalline polymers \\
\hline Keywords (user supplied): & chevron, folds \\
\hline
\end{tabular}

\section{今 scholarONE" \\ Manuscript Central}




\title{
Chevron morphology in deformed semicrystalline polymers
}

\author{
M. KRumova*, S. HenNing, G. H. MichleR \\ Martin-Luther-University Halle-Wittenberg \\ D-06099 Halle (Saale), Germany
}

\begin{abstract}
Chevron morphology was observed using transmission electron microscopy in various semicrystalline polymers deformed in tensile experiments. The morphological and mechanical prerequisites for chevron structure formation in semicrystalline polymers were revealed. It was demonstrated that chevron folding is a common deformation mode which can appear in real, i.e. globally unoriented or partially oriented samples, in areas where the lamellar stacks are oriented perpendicular to the deformation direction. Similarities with the behaviour of other layered systems were found. The mechanisms of chevron formation is discussed in the light of the fundamental statements of the folding theories and is related to the specific microstructure of the polymers. The effect of the boundary conditions, deformation temperature and macroscopic strain on the characteristics of the chevron structure is described.
\end{abstract}

Keywords: chevron, folds, semicrystalline polymer, deformation, morphology, electron microscopy

1

*Author for correspondence. E-mail: marina.krumova@iw.uni-halle.de 


\section{§ 1. INTRODUCTION}

Folded structures can be often observed in layered materials at a great diversity of length scales. The earliest investigations on this phenomenon date back over a century and were performed in the field of the structural geology. Natural folds in rocks were studied in details and different types (sinusoidal, concentric-like, chevron, kink and asymmetric folds) were distinguished and classified. On the bases of these observations and experiments with model systems, theories about the mechanisms and mechanics of folding have been developed. There is an extensive body of literature devoted to the practical experience and theoretical treatments of layer folding. Some geology textbooks, e.g. $[1,2]$ offer a detailed review of the most important results

Folds comprising straight limbs and sharp hinges are known as chevrons. Chevron patterns can be found in metamorphic, volcanic, and sedimentary terrains in many parts of the Earth. They range widely in scale, with wavelengths from a few $\mathrm{mm}$ to about $1 \mathrm{~km}$ [1].

Formation of chevron morphology at a scale of about $100 \mu \mathrm{m}$ has been observed later in thin magnetic films, comprising stripe phases as a pattern of self-organisation. When the stripe phases are strained by changing either the temperature or the applied magnetic field in a direction tending to decrease the equilibrium stripe spacing, the stripes buckle in order to bring the local layer spacing closer to equilibrium [3,4]. The initial modulation appears above a certain strain threshold and is sinusoidal. With increasing strain the stripe modulation pattern incorporates higher Fourier modes and takes a zigzag or chevron appearance [5].

Chevron folding occurs even at molecular length scale - in liquid crystals confined between the flat parallel boundaries of the solid cell. The buckling phenomena has been observed in the homeotropic geometry, when the smectic layers are parallel to the plates [6], as well as when they are perpendicular to them, in "bookshelf" geometry [7]. The chevron is believed to be a consequence of the mismatch between the natural thermodynamically stable smectic layer thickness and that imposed by layer pinning at the cell surface [8]. The undulation is generated either by explicit tension perpendicular to the layers or simply by changing the temperature. The wavelength is imposed by the cell size and usually amounts some micrometers.

In the mentioned layered systems folding occurs as a response to deformation force acting perpendicular to the layering, The phenomenon seems to be based on the same fundamental mechanisms in very different materials, on very different length scales ranging from macroscopic geological formations to the molecules of smectic liquid crystals. 
Chevron pattern attracted again scientific attention with the study of the deformation behaviour of thermoplastic elastomers, such as styrene-isoprene-styrene or styrene-butadienestyrene copolymers. It was found that if SIS or SBS copolymers in a globally aligned hexagonal or lamellar phase are stretched perpendicular to the rods or plates, then above a critical strain the lamellae or cylinders buckle to form a chevron or zig-zag structure. The morphology was detected and characterised by different investigation techniques, SAXS [9, 10], combined SAXS measurements and transmission electron microscopic (TEM) observation [11, 12], TEM [13]; Fourier transform infrared (FTIR) spectroscopy [14] and Scanning force microscopy (SFM) [15]. In a recent work [16] Read et al. modeled the instability towards chevron folding in layered block copolymers in terms of the macroscopic elastic moduli of the material, making corrections to account for microscopic deformations caused by the buckling.

The morphology of the majority semicrystalline polymers exhibits alternating crystalline lamellae and interlamellar amorphous spaces and thus approaches a nanoscaled layered system, even if with lower perfection. Consequently, upon loading perpendicularly to the lamellar stacks, formation of chevron structure can be expected. Actually, chevron structure has been observed in some polymers as a result of special processing, in the aim to produce single crystal-like texture. In the 1970s, various polymers have been subjected to cold-rolling and the structural changes which occur during rolling have been studied in detail. Chevron structures have been observed by TEM in polyoximethylene (POM) [17] and polyethyleneoxide (PEO) [18], and called "banded zig-zag type of lamellar structure with the bands parallel to the roll direction". Later, chevron like morphology has been found in high density polyethylene (HDPE), again as a result of special processing, rolling and channel-die compression, e.g. [19, 20], melt-drawing [21] or film blowing, e.g. [22]. All these investigations were predominantly concerned with lamella tilting as a result of the processing, the strange "zig-zag" morphology was not attributed to a special deformation mode. Analogy with similar structures in other systems was not realised.

Only few works describe the formation of this morphology in traditional tensile experiments. The materials studied were HDPE and UHMWPE [22-25]. However, neither in these publications the term "chevron" was used, the morphology was either mentioned without any denomination or was called "herring-bone" or "fir tree-like".

Recently a study focused on the mechanism of polymorphic transformation of $\beta$-PP into $\alpha$-PP upon deformation has been published [26]. On some of the TEM micrographs, presented in the publication, chevron morphology can be easily recognised. The authors, however, mention this behaviour as shearing and tilting of broken horizontal lamellae towards the loading direction. 
In the last years we repeatedly found chevron pattern in other polymers deformed in traditional tensile experiments. Our observations for polypropylene we presented in recently appeared publications [27-29].

In this report we are not going to present the result of one particular investigation, rather we wish to summarise the material accumulated over the past years in our laboratory. Further in this work we aim:

(1) to extend and generalise the experience on this phenomenon in semicrystalline polymers

(2) to demonstrate that chevron folding is a common deformation mode which can appear in a real, i.e. globally unoriented or partially oriented semicrystalline polymer in regions where favourable conditions exist

(3) to compare and correlate the main characteristics of chevron pattern formation in semicrystalline polymers with the existing theories on mechanism and mechanics of folding in other layered systems.

\section{$\S 2$. EXPERIMENTAL DETAILS}

\subsection{Materials}

For this investigation we selected materials, which represent different classes of semicrystalline polymers.

Polypropylene (PP) was chosen as a material from the family of commodity plastics. Samples were prepared from high molecular weight commercial isotactic polypropylene grades (Borealis AG): $\alpha$-iPP was Daplen BE50 and $\beta$-iPP was Daplen BE60. Sheets with thickness of 1 $\mathrm{mm}$ were produced by compression moulding and subsequent multistage crystallisation procedure in the second $\beta$-iPP case.

Syndiotactic polystyrene (sPS) is a relatively new material and was interesting for our study due to its high glass transition temperature, so that deformation at common conditions (room temperature) practically takes place with amorphous phase in glassy state. The used sPS was commercial grade (Dow Chemical Corp.) with molecular weight $220000 \mathrm{~g} / \mathrm{mol}$ and syndiotacticity of $98 \%$. Plates of $1 \mathrm{~mm}$ thickness were prepared by compression moulding and crystallisation at $245^{\circ} \mathrm{C}$ to obtain the $\alpha$-sPS modification and by slow cooling to obtain the $\beta$-sPS modification. We report here for the first time about formation of chevron morphology in sPS upon deformation. 
In addition we used results of previous investigations of HDPE and UHMWPE, where chevron formation was first found in our tensile experiments. Some of these results are reviewed in the book on micromechanics in polymers by Michler [30].

\subsection{Experimental Techniques}

\section{Mechanical Testing}

Macroscopic tensile deformation experiments were carried out using universal tensile machine Zwick 1425 (Ulm, Germany) and a MINIMAT miniature materials testing device (Polymer Laboratories, UK). Standard tensile bars were prepared from the pressed sheets for the Zwick machine. For the MINIMAT device miniaturised, dumbbell-shaped tensile bars were punched out of the plates using a special pierce tool. Tensile deformation experiments were performed at a cross-head speed of $1 \mathrm{~mm} / \mathrm{min}$ at various temperatures. The strain rates in both cases were of the order of $2.10^{-4} \mathrm{~s}^{-1}$.

\section{Transmission electron microscopy (TEM)}

Ultrathin sections of about $70 \mathrm{~nm}$ were prepared from regions close to the middle of the tensile bars, corresponding to different states of elongation. The sections were cut parallel to the tensile direction by means of a Leica Ultracut microtome equipped with a Diatome diamond knife. For contrast enhancement the materials were stained with $\mathrm{RuO}_{4}$ vapour. Micrographs were acquired using a JEOL transmission electron microscope JEM 2010, operated at $200 \mathrm{kV}$ accelerating voltage. On the micrographs the crystalline lamellae appear bright and the amorphous regions dark.

\section{$\S 3$. RESULTS AND DISCUSSION}

\subsection{Prerequisites for chevron pattern formation in semicrystalline polymers}

On the bases of a great amount of results obtained by direct imaging of microdeformation structures in various polymers, we can conclude that not in each polymer chevron pattern are formed upon deformation. Some prerequisites that enable the chevron folding must be present. 
Morphology

As a first prerequisite we consider the morphological one, that is the existence of stacks of nearly parallel crystalline lamellae, i.e. layered arrangement, as for example, in the case of the $\beta$-polymorphic modification of isotactic polypropylene ( $\beta$-iPP), shown on Figure $1 \mathrm{a}$. The lamellae in this arrangement are able to undergo cooperative folding (Figure 2) when dilative force is applied normal to the layers. A great difference exists between this morphology and that of the more common $\alpha$ polymorphic modification of iPP, as evident from Figure $1 b$. Characteristic for $\alpha$-iPP is the so called "cross-hatched" arrangement of the crystalline lamellae. The main lamellae ("parent lamellae") are growing radially from an initial site (centre of the spherulite), and "daughter lamellae" are formed by an epitaxial growth onto them at an angle of about $81^{\circ}$. In this way a kind of network is formed, not prone to chevron folding during deformation.

Morphological differences present also high (HDPE) and low (LDPE) density polyethylene. The disordered lamellar arrangement of LDPE (Figure 3) does not favour chevron formation upon deformation. In contrast, HDPE forms stacks of parallel lamellae, which undergo rotation and folding when perpendicular force is applied. This behaviour has been shown in [23] and [25] for HDPE-blow foils upon additional drawing (Figure 4). Similar behaviour has been reported also for ultra high molecular weight polyethylene UHMWPE after straining [24].

Analogical situation offers the comparison of isotactic (iPS) and syndiotactic (sPS) polystyrene. In iPS the lamellae are curved as a result of the lamellar stacks growth outward from the central regions of the spherulites by splaying and branching [31,32] Thus, the stack can hardly be regarded as a nanolayered system of nearly parallel layers. In contrast, the lamellar arrangement of sPS shown on Figure 5, looks very similar to that of $\beta$-iPP, represented on Figure 1 a.

Nevertheless sPS does not form chevron patterns when deformed at room temperature, this means that the morphological prerequisite is not sufficient for chevron formation.

\section{Alternating hard and soft layers}

The concept of alternating hard and soft layers emerges in the earliest investigations on folding. It has been realised that for fold formation slippage between layers and flowage of material from limbs toward crests and troughs is needed, i.e. angular folds can develop when the layers have markedly different physical properties. Layers of different mechanical compliances show different resistance to the applied deformation stress, they react and adjust their strain by 
buckling at a point of instability. The soft layers play an important role: they start to deform plastically and ensure the strain needed for initiation of the bending of the hard layers.

In polymer physics the condition of alternating hard and soft layers is well illustrated with the deformation behaviour of block copolymers with lamellar morphology. Styreneisoprene-styrene and styrene-butadiene-styrene copolymers comprise alternating glassy polystyrene and rubbery butadiene or isoprene lamellae and form chevrons upon deformation at room temperature. Differently, in styrene - methyl methacrylate block copolymers, with lamellar morphology, both phases are hard (in glassy state) at room temperature. Stretching provokes in this case crazing and not chevron folding [33].

The studied semicrystalline polymers (HDPE and $\beta$-iPP) can be regarded as layered systems of hard crystalline lamellae and soft amorphous interlamellar layers. When deformation is applied at room temperature, the amorphous phases of these polymers are really soft, since being well above their glass transition. The same holds also for the above mentioned POM and PEO, in which chevron structure has been observed as a result of cold rolling (see $\S 1$. Introduction). Due to their very low glass transition temperatures of $-83^{\circ} \mathrm{C}$ and $-67^{\circ} \mathrm{C}$, respectively, the amorphous interlamellar regions are soft when deformation is performed.

Differently behaves a semicrystalline polymer with glass transition above room temperature. The syndiotactic polystyrene (sPS) we chose as a model for such polymers, fulfils the morphological prerequisite (see Figure 5). But due to its high glass transition temperature of nearly $100^{\circ} \mathrm{C}$, the amorphous layers are far from being rubbery, the mobility is restricted and the material deforms at room temperature in a brittle manner. Only after raising the deformation temperature above $100^{\circ} \mathrm{C}$, the required mobility (rubberlike state) is reached and chevron pattern appear in corresponding areas, as demonstrated on Figure 6.

From these observations the second prerequisite can be deduced, i.e. a large difference in the mechanical and/or thermal properties of the crystalline and amorphous phases of the polymer.

The condition of difference in the physical properties of the two type of layers has been extensively discussed in the literature on folding. The difference between hard and soft is expressed in terms of stiffness, elastic modulus, compliance, mobility, viscosity, glass transition temperature etc., depending on the multilayer and on the model used for investigation of the phenomenon. In the geology usually viscosity difference is considered. It has been shown that even the fold shape depends on the viscosity ratio of the two types of layers [2]. In multilayers an additional factor is also important in controlling fold shape. It is found that, for any two materials of particular ductility contrast the curvature of the fold surfaces is strongly affected by the 
proportion of softer to stiffer material (the $n$-value). With a decrease in the proportion of softer material high curvatures are concentrated near the hinge zone and the fold limbs become less curved, so the overall shape approaches more closely the sharp-hinged, parallel-limbed chevron fold [2]. At moderate $n$-value, which is relevant for the case of semicrystalline polymers, the formation of chevron folds is favoured at high viscosity (ductility) contrast. The question about a "threshold contrast" is more complicated. It can be evaluated for each particular multilayer using the mathematical expressions for the initial wavelength of folding derived from the theoretical treatments. Some of these expressions will be discussed in more details in $\S 3.4$.

In polymer physics predominantly Young's-modulus difference of the two type of layers is considered. Finite element modelling of the folding process in styrene-butadiene block copolymers with lamellar morphology has followed the development of the fold shape at different E-modulus - ratios [16]. In a single layer chevron pattern is observed to form at an Eratio of 100 , in a multilayer, with the particular parameters selected, first sinusoidal folds develop at E-ratio of 500 between stiff and soft layers.

Generally, in styrene-butadiene block copolymers a large difference between the Young moduli of PS and PB exists, with a ratio up to 1000 times. In the case of semicrystalline polymers as a modulus of the amorphous phase the value of the rubbery plateau can be considered, which usually amounts some decades MPa. Then, the ratio between the moduli of crystalline and amorphous phases will be of the order of x1000 to x10 MPa.

\subsection{Mechanism of chevron folding in semicrystalline polymers}

A real sample of a semicrystalline polymer between the grips of the tensile stretching device is usually globally unoriented or only partially oriented, comprising lamella stacks randomly distributed in various directions. Schematically such material can be represented as consisting of regions (domains) of parallel lamellae, the domains themselves situated at different angles with respect to the deformation direction. (Figure 7a).

All three currently established local deformation modes can take place in the lamellar stacks in these domains. Whole stack (domain) can rotate if it is surrounded by amorphous matrix and hence, free to rotate under the stress. In the domains comprising lamella stacks at some angle to the stretching direction interlamellar shear is the most probable deformation mechanism. It involves slip of the lamellae parallel to each other with the amorphous phase undergoing shear, which is a relatively easy deformation mechanism for a polymer above the 
glass transition temperature [34]. With stretching, the stacking axes of the lamellae in these domains get closer to the stretching direction, this enables longitudinal extension together with lateral contraction of the domains.

The object of interest for this investigation are the domains comprising lamella stacks perpendicular to the stretching direction, as the central domain " $\mathrm{A}$ " in the scheme of Fig. 7a. According to the established local deformation modes this domain initially mostly deforms by interlamellar separation. As known, the amorphous regions are entangled networks solidly attached to the adjacent lamellae, the lamellae are hold together by tie molecules through the amorphous phase. At the beginning of macroscopic stretching, the lamellae are displaced rigidly, and the ensuing interlamellar separation leads to strong hydrostatic tension within the constrained amorphous network. The tie molecules become more extended and transfer locally concentrated elastic stresses to the lamellae [35]. Stress relief can occur in two ways, through cavitation within the amorphous network and/or through crystal plastic deformation.

At the same time this domain of parallel lamellae can be regarded as a multilayer subjected to a deformation force perpendicular to the layering. Additionally, a contraction stress component is acting parallel to the layering due to the plastic deformation of the neighbouring domains (macroscopically expressed as the Poisson ratio). Then, like other layered systems, after reaching a critical strain this lamella stack should undergo folding. Qualitatively this behaviour can be explained with the aim of the lamella stack to recover the equilibrium spacing and not to change significantly the volume, similar to the mechanism established for other layered systems (see $§ 1$. Introduction). According to theoretical treatments of deformation in multilayers, folds (angular or rounded) may result from the natural tendency of materials to deform in energy minimising way [36]. These theoretical treatments have further demonstrated that angular folds (chevrons, kinks etc.) have the most economic profile as regards energy consumption. Recently, Cohen et al. [15] demonstrated that also in SBS block copolymers with lamellar morphology bending of the hard layers is less costly in free energy than dilation of the layers, which results in folding of the layers by kinking or undulation into chevron morphology.

In semicrystalline polymers the situation may be more complicated. As mentioned above stress relief can occur in two ways, through cavitation within the amorphous network and/or through crystal plastic deformation. Due to the great number of defects (such as chain ends or weak entanglements) cavitation in the amorphous regions can easily occur. Under tensile stress both processes can be observed: i) chevron folding, which involves lamella plastic deformation and damps microvoid formation or/and ii) lamella separation with cavitation. One of these 
concurrent processes or both simultaneously will take place in dependence on the specific characteristics of the polymer.

It is established that plastic deformation of polymer crystals is generally crystallographic in nature, the main mechanism being crystallographic slip, twinning and martensitic transformation. The slip mechanism is the most important since it can produce larger plastic strains. Slip takes place when the resolved shear stress on the slip plane reaches a critical value, known as the critical resolved shear stress. Cavitation during deformation can be observed in polymers for which the critical resolved shear stress for the easiest slip system of crystals is higher than the stress required for cavitation [34]. In the other cases, crystallographic slip will occur earlier, relaxing the stress and a cavitational pore will not appear. Hence, it can be expected that in latter polymers chevron folding will be facilitated at low strains.

Our experiments on HDPE show only chevron folding and no cavitation upon straining perpendicular to the lamellae stacks. This behaviour can be explained with the relative low values of the resolved stress for the easiest slip (shift in chain direction) in HDPE, between 7.2 and 15.6 MPa [20, 37]. On the other hand, it has been shown that the values for the resolved stress depend also on the crystal thickness, so that cavitation can be found in HDPE slowly cooled or formed in thick wall products [34]. The HDPE investigated in our laboratory comprised relatively thin lamellae, of the order of $15 \mathrm{~nm}$. In HDPE samples with thicker lamellae [21] simultaneous kink formation and cavitation of the amorphous regions are observed upon tensile deformation.

In $\beta$-polypropylene chevron pattern and microvoids can be simultaneously observed in deformed samples, as illustrated in Figure 8. Consequently, both competing processes take place. According to Galeski [34] $\alpha$-polypropylene belongs to the polymers in which cavitation during deformation can be expected, due to its relative high value of the resolved stress for the easiest slip (in chain direction), around 22-25 MPa. Unfortunately, there are no data about this stress in $\beta$-PP available yet in the literature. Based on the facility for chevron folding in $\beta$-polypropylene we could suppose that the critical resolved stress for the easiest slip in $\beta$-PP is lower than in $\alpha$ PP and comparable with the critical stress for cavitation.

It should be mentioned that the critical resolved shear stresses for slips are well known only for few polymers. They were measured using samples of rather well defined texture. As expected this parameter is yet unknown for the relatively new syndiotactic polystyrene (sPS), which shows chevron morphology upon stretching (see Fig.6). The frequent appearance of 
chevrons and the absence of voiding in this polymer could be an indication of relatively low critical resolved shear stresses for slips in its lamellae.

Beside the resistance to plastic deformation of the crystal phase, the constraints on the amorphous phase have to be taken in account. In the vicinity of the glass transition a higher quantity of voids can coexist with chevron folds due to the reduced mobility and deformability of the amorphous network.

\subsection{Critical strain}

It is well established that the initial layered structure transforms to chevron morphology beyond a critical strain. Of practical importance would be a relation of this transformation to changes in the stress-strain behaviour. However, in our case of globally unoriented semicrystalline polymers this issue is difficult to be resolved. We can only estimate the local strain in the vicinity of the domain which forms chevrons and indicate the approximate region of the macroscopic stress-strain curve, where chevron formation can be expected. Some qualitative considerations for the upper strain limit can be deduces in addition from works on morphological changes upon deformation which are focused on the onset of fibril formation.

More attention is paid on this problem in block copolymers with nearly perfect lamellar arrangement of the hard and soft phases. Since they can be regarded as a model of nanolayered polymer, we shall make use of some results from these investigations. Among the published works two main views can be distinguished. Some authors associate the critical strain with the yield point and predict layer folding at very low strains of only some percent $[16,38]$. Others [e.g. 15] found that deformation perpendicular to the layers proceeds at room temperature uniformly without any noticeable necking and the four-point SAXS pattern, characteristic for chevron structure appears only beyond the yield point when the stress-plateau is reached. In other works on globally oriented SBS block copolymers values for the critical macroscopic strains of about 30 or $40 \%$ are reported [9, 13, 39]. Interesting for us is an investigation of globally unoriented SBS samples [10]. Chevron morphology formation was observed there at about $20 \%$ strain.

Our findings for unoriented semicrystalline polymers correlate well with the latter results. SEM and TEM micrographs of $\beta$-PP deformed at room temperature show formation of chevron pattern at about $20 \%$ local deformation. In the above cited work on polymorphic transition of $\beta$ PP upon deformation [26] a TEM micrograph shows chevron pattern at local strain of about 
$35 \%$. We suppose that chevron morphology has appeared at smaller deformation, but the corresponding pictures are not shown, because the attention in the study is focused on other structures.

In sPS we performed the deformation at higher temperature for the reasons explained in $\S$ 3.1. We observed formation of chevron morphology also at roughly $20 \%$ local strain. The corresponding stress-strain curves did not show any noticeable necking. [40].

We have not done specific investigations for measurement of the critical strain in HDPE beyond which chevron pattern appear. Nevertheless on the basis of works devoted to the general deformation behaviour of HDPE, some estimations can be made. In early studies, dating back over 30 years, it has been found that the critical strain level for texture developing in polyethylene upon deformation at room temperature appears to be near $20 \%$ [41]. Subsequent detailed investigation on the deformation behaviour in spherulitic PE by Petermann and Schultz [42] has shown that upon tensile straining at low deformation rates PE behaves reversibly for extensions up to $40 \%$. Higher elongation is needed for initiation of the other deformation modes which are mainly plastic, as slip, chain kinking etc. Other investigations dealing with the deformations in PE also associate the onset of plastic deformation modes with macroscopic elongation beyond 20\%. Analysing the transition points in the true stress-true strain curve Strobl et al. [43] associated the Hencky strain region between $\varepsilon_{\mathrm{H}} \approx 0.1$ and $\varepsilon_{\mathrm{H}} \approx 0.6$ (corresponding to nominal strains between 10 and 80\%) in PE with collective slip processes. They also suggested that the "coarse" block slip processes should be dominant. Up to $\varepsilon_{\mathrm{H}} \approx 0.6$ the lamellae, although set together of displaced blocks, still exist. If we make use of this schema, we can expect chevron folding to occur beyond $10 \%$ and begin to disappear beyond $80 \%$ nominal strain.

It is interesting to remind here some observations from cold rolling of polymers. Rolling is similar to drawing in that the tangential (frictional) force of the roller provides a component which exerts a tensile stress on the material. Therefore, rolling can be considered to be a combination of drawing along the roll direction and compression along the thickness direction. In rolled POM [17] chevron like morphology has been observed at extend of the roll $\mathrm{L} / \mathrm{L}_{0}=0.8$ (i.e. deformation of about $20 \%$ ).

Summarising all these results, it appears that chevron formation in semicrystalline polymers can be associated with the region of the stress-strain curve beyond the yield point, when the stress-plateau is reached. At the low strain rates used in these experiments, the critical strain varies with the material roughly between 15 and $40 \%$.

From microstructural point of view, the crystalline lamellae start to deform plastically (slip is initiated) after the entangled non-crystalline chains have reached their elongational limit. 


\subsection{Characteristics of the chevron morphology and correlations}

In the literature dealing with chevron morphology the following main characteristics are discussed: wavelength, layer spacing and the tilt angle of the layers with respect to the stretching force. In the following we will make an overview of the chevron structures observed in the studied polymers and describe their changes with deformation temperature and strain.

\section{Wavelength}

From the evaluation of the great amount of micrographs showing chevron morphology in the studied semicrystalline polymers we can conclude that the typical size of the regions (domains) undergoing chevron folding lies between 1 and $2 \mu \mathrm{m}$ and that in average 3-4 folds are accommodated in these regions. The wavelength varies between 200 and $500 \mathrm{~nm}$ with material, strain and deformation temperature. Often folds with different wavelengths can be found in one sample, as the enclosed by the circles in Fig. 9. The chevron folds are not always symmetrical, 
often one arm ("limb") is longer than the other. The micrographs show further that symmetrical and asymmetrical folds can coexist in one sample, in different domains. In the case of $\beta$-PP, for example, this can be seen comparing Fig. 2 and Fig. 8.

All these peculiarities can be explained using the results of the general theories of folding developed for other layered materials. Now the mechanism of undulation instability is prevalently accepted for the response of layered materials to deformation perpendicular to the layers. According to it above a critical stress the multilayered materials buckle, with an initially sinusoidal buckling profile.

The undulation instability is a highly cooperative phenomenon of layers between confining external boundaries. The boundary conditions force adjacent layers to maintain their parallel orientation and limit the ability to undulate with a long wavelength. In this way the sample distance between the imposed boundary conditions is important in determining the wavelength of the buckling. The stable wavelength in the multilayered stack is affected further by the average properties of the material of the boundary (or outside of the stack). In the case of liquid crystals the boundaries are the parallel flat walls of the cell. In a perfectly aligned layered polymer the correlation distance for which the layers are coherently packed, could be in first approximation the macroscopic distance between the grips of the tensile stretching device. However, it is in practice very difficult to obtain in polymers such an ideal macroscopic arrangement. As mentioned in $\S 3.2$. a real sample, globally unoriented or partially oriented, can be schematically represented as consisting of domains of parallel lamellae, the domains themselves situated at different angles with respect to the direction of deformation (Figure 7). Chevron folding is completed only in domains with lamellar orientation perpendicular to the applied force. Consequently, the boundary conditions in this case will be imposed by the surrounding domains. Evidently, the boundary conditions are different for each domain which undergoes chevron folding. Moreover, the boundary conditions also change in the process of deformation.

The wavelength of the chevron profile is sensitive to both, to the boundary conditions on the multilayered material, and to the details of its structure. Mathematical expressions for the dominant wavelength of the folds which can develop in a system under external stress have been derived from the theoretical descriptions of the origin of folding. Most of the theoretical research has been devoted however to understanding of folding of single layers embedded in a surrounding medium, so that the theory of single-layer folding is quite highly developed. In the case of geological folding (a general solution for viscoelastic materials) the initial fold 
wavelength is shown to be controlled by layer thickness and viscosity contrast $[1,2]$. The following equation for the initial (sinusoidal) wavelength $\lambda$ is currently accepted:

$$
\lambda=2 \pi d\left(\sqrt[3]{\mu_{h} / 6 \mu_{s}}\right)
$$

where $\mu_{h}$ and $\mu_{s}$ are the viscosities of the hard and soft layers respectively, and $d$ is the thickness of the hard layer.

A linear dependence of the critical (initial) wavelength on the layer thickness has been derived also in the engineering mechanics for buckling of a single beam (layer) supported by elastic foundation [45]. Recently the linear dependence has been proved experimentally also for a 3-layer polymer system (elastomer-plastic-elastomer films) [46]. The following relationship involving the elastic moduli $E_{P}$ and $E_{R}$ of the plastic and rubbery layers has been proposed, which is more practical for calculations in the case of semicrystalline polymers than (1):

$$
\lambda=\pi d\left(\sqrt[3]{4 E_{P} / 3 E_{R}}\right)
$$

In multilayers these relationships are getting more complicated due to the great variety of possible combinations of material properties, layer thicknesses and interrelations between the two types of layers. In the simplest multilayer model of an infinite stack of regularly alternating layers the development of folds is controlled also by the viscosity contrast, but instead of the thickness of the stiffer layer, by the proportion $n$ of softer to stiffer layer thickness [2]. When $n=$ $\infty$ the solution coincides with the well defined initial wavelength developed in a buckled single stiff layer in softer medium deduced from equation (1). As the stiffer layers come to lie closer together (decreasing $n$-value), for a given stiff layer thickness, the characteristic initial wavelength becomes larger.

The features of the folded structure we observed in the studied semicrystalline polymers are in good agreement with the above model. An approximated calculation of the wavelength $\lambda$ of a buckled single lamellae according to equation (2), gives for typical lamella thicknesses in the range $10-15 \mathrm{~nm}$ values for $\lambda$ between 250 and $400 \mathrm{~nm}$. In the folded lamella stacks we always measured larger wavelengths for the respective polymers.

The exact mathematical expressions for the initial wavelength of folding multilayers, even composed of identical stiff layers and identical soft layers are very complicated. The wavelength defined according to the elementary theory of folding (the Ramberg wavelength) [1]

$$
\lambda_{R}=2 T \sqrt{\pi m / N} \sqrt[4]{\left(B_{1} / 12 B_{v}^{\prime}\right) /(1-D)}
$$


includes in addition dependence on the total thickness $T$ of the multilayer and the number of pairs of layers $N$. $B_{v}$ and $B_{v}{ }^{\prime}$ are calculated in terms of the layer thicknesses $t_{i}$ and moduli $B_{i}$ according to:

$$
\begin{aligned}
& B_{v}=\left(t_{1}+t_{2}\right) B_{1} B_{2} /\left(B_{1} t_{2}+B_{2} t_{1}\right) \\
& B_{v}^{\prime}=B_{v}\left(1+t_{2} / t_{1}\right)^{3}
\end{aligned}
$$

Here $i=1$ refers to stiff and $i=2$ to soft layers and:

$$
B_{i}=E_{i} /\left(1-v_{i}^{2}\right)
$$

where $E$ is Young's modulus and $v$ is Poisson's ratio.

The factors $m$ and $D$ are interrelated in a complex manner and must be determined iteratively. Graphical solutions to these equations for various thickness and moduli ratios can be found in the literature. We used them for an approximate estimation of the expected initial wavelength of the folds in our semicrystalline polymers.

According to the TEM micrographs the thickness ratio of amorphous layers to crystalline lamellae vary between 1 and 2 . For modulus ratio $B_{2} / B_{1}$ we adopted the approximate value of 0.01 and calculated $B_{v}$ and $B_{v}{ }^{\prime}$ after eq. (4) and (5). As mentioned above the typical size of the domains we found to undergo folding in our polymers lies between 1 and $2 \mu \mathrm{m}$, this corresponds to the total thickness $T$ of our multilayer; and the number of pairs of layers $N$ is the ratio of $T$ to the long spacing. Thus $N$ varies in the studied polymers in the range from about 60 to 200 . Using these values we determined the ratio $\lambda / T$ from the graphical solutions for (3), presented in Fig.4, p.385 of [1]. We obtained results of $0.2-0.3$ in average, which are in a very good agreement with our experimental observations.

It should be mentioned that equations (1) and (3) describe the values of the wavelengths of the initially formed folds, which have sinusoidal form. Nevertheless, the use of these equations for the wavelength of chevron folds is also justified. It is established that with increasing strain the undulations evolve into chevron shape. The linearized theory of folding, proposed by Johnson [1], describes mathematically this transformation of the initial sinusoidal folds trough concentric-like to chevron folds and shows that the wavelength is related to the dimensional and rheological factors approximately as shown in eqs. $(3-6)$. The sinusoidal pattern becomes modified by high order effects, but the wavelengths of the final chevron folds, measured along layers, is roughly equal to the wavelength of the initial sinusoidal folds.

In the globally unoriented polymer samples domains (stacks) of different sizes and correspondingly different numbers of layers can be found. From the above relations it can be deduced that folds can form simultaneously in several domains, if they contain the same number 
of layers with identical elastic properties. But the wavelengths would be different if the thickness of individual layers were different in the separate multilayers. The wavelengths can be different also when individual layer thickness is the same, but the number of layers in separate domains differs. In semicrystalline polymers these structure details are the degree of crystallinity, lamella thickness and elastic characteristics of the crystalline and amorphous phase of each polymer. Obviously, in a real sample variations in lamella thickness and length always exist, as well as in number of layers in separate domains. Thus, folds with different wavelengths can be formed in one semicrystalline sample.

The coexistence of folds with different wavelengths, as illustrated in Fig. 9, can be also explained as a consequence of the equal probability for fold formation with different wavelengths. According to the theoretical modelling, with closing together of the stiffer layers (decreasing $n$ ), initial fold wavelengths over a wide range of values are equally favoured and the initial wavelength in the multilayer can become very irregular [47]. For the semicrystalline polymers studied, with their moderate to low value of $n$, typically about 1 , these results could be valid. At some conditions the folds which are most stable have the largest wavelength possible in the system, probably governed by the mechanical imperfections in the multilayer. From aspect of the semicrystalline microstructure these imperfections are related to local variations of the degree of crystallinity and lamella thickness, to defects in the crystalline structure and variations in density and length of the tie molecules connecting adjacent lamellae.

On the other hand chevron pattern with similar wavelength and amplitudes can be observed on the micrographs, although the number of lamellae and their thickness are not equal in all domains. This fact can also find its explanation in the graphical solutions to the equations of folding. According to them, appropriate combinations of the dimensional and rheological properties can result in similar wavelengths for very different multilayers [1].

An important peculiarity of the chevron morphology in semicrystalline polymers is the asymmetry of the folds. Asymmetry often exists also in geological formations. There it is explained as a result of interactions of folds with different wavelengths in the process of formation. The probability of such mechanism can be associated with the inhomogeneous initial structure of the semicrystalline polymers. In a real sample a perfect alignment of the lamellae parallel to each other is impossible. The lamellae are growing usually radial from an initial site (centre), so that certain curvature is inevitable. Further, in a real sample a distribution in lamellar thickness always exists, as well as different kind of defects. It may well be that these variations, lamellar waviness, dislocations and defects provide another length scale of folding in some regions. In the preceding paragraph the important role of the tie molecules for initiation of the 
lamella plastic deformation was pointed out. In a real sample obviously a density and length distribution of the tie molecules exists and this inhomogeneity can additionally affect the local folding.

In this way the asymmetry can be understood still accepting that chevrons are formed by undulation instability. On the other hand, the distortions of the chevron shape can be also explained if an alternative mechanism of chevron formation is supposed. Theoretical treatments and geometrical analysis have demonstrated that chevron folds can result from interaction of conjugate kink folds [48]. Recently, Cohen et al [12, 15] assumed in their investigations on deformation of SBS block copolymers that kink bands first nucleate around defects and the further propagation of the kink boundaries into adjacent regions leads to chevron morphology.

Such mechanism is conceivable for semicrystalline polymers due to the great amount of defects in their multilayers. Nevertheless, we did not find in the polymers studied any indication of this mechanism. At the deformation conditions used in our investigations we did not observe kink formation, neither coexistence of kink bands with chevron folds. It should be mentioned however, that we have not followed the deformation process "in situ". The observations in TEM are made after the deformation is completed, so that the final result of the deformation is imaged. If we assume that the chevron folding is initiated by kink nucleation, we should further suppose a very fast transformation of the kinks to chevrons in the whole volume of the domain of interest. Some more details about this problem are discussed below, in $\S$ Tilt angle.

\section{Layer spacing}

Even the earliest studies of chevron profiles in rocks found that harder beds (layers) maintain roughly constant thickness whereas the softer layers thicken in the hinges. The analytical treatments of the transition from sinusoidal undulations to chevron morphology applicable to layered geological structures [36] and to smectic liquid crystals [5] and striped magnetic patterns [4] also predict changed in the layer spacing with deformation.

For the investigation of this process in thermoplastic block copolymers and rolled semicrystalline polymers predominantly SAXS and electron microscopy were used. It was concluded that in SBS block copolymers with layered morphology the long spacing remains nearly constant in the straight limbs of the chevron folds, but in the hinge regions the soft PB layers are more dilated than the PS ones. Our observations on chevron morphology in deformed SBS block copolymers (Figure 10) also support this statement. 
The electron microscopy investigations we performed of the chevron structure in semicrystalline polymers show also some thickening of the dark layers (corresponding to the amorphous phase) in the hinge regions, see Figs. 2, 3, 6. Consequently, the general features of the chevron structure in layered systems are present also in the folded domains of deformed semicrystalline polymers. This thickening of the amorphous phase in the hinge regions can be better distinguished when the difference between test temperature $\mathrm{T}_{\text {def }}$ and glass transition temperature $T_{g}$ of the polymer is larger. Figure 4 illustrates the larger dilation in the hinge region in HDPE, compared with $\beta$-PP (Fig. 2) and sPS (Fig.6); the latter deformed at only $10^{\circ} \mathrm{C}$ above its $\mathrm{T}_{\mathrm{g}}$.

\section{Tilt angle}

The tilt angle $\varphi$ is defined as the acute angle that the limb of the chevron fold makes with the deformation direction. It represents the degree of tilting of lamellae and shows how sharp the chevron pattern is. In earlier works the interlimb angle $\psi$ is used, corresponding to twice the tilt angle, i.e. $\psi=2 \varphi$. The tilt angle changes with the strain and the temperature at which the deformation is performed. We followed this changes performing deformations of the semicrystalline polymers at different temperatures.

According to the theories of undulation instability above a critical stress the multilayered materials buckle, with an initially sinusoidal buckling profile which further transforms to chevron pattern. TEM do not allow to observe the buckling process "in situ". The micrographs show the final result after the deformation is completed. In order to check if this mechanism applies also for semicrystalline polymers we tried to perform investigation at conditions near to the critical ones, where the undulation starts.

For a semicrystalline polymer a critical region is around the glass transition $\mathrm{T}_{\mathrm{g}}$, since below $T_{g}$ folding is hindered, according to the discussion in $\S 3.1$. Thus we studied the deformation structures in SPS at temperature of $100^{\circ} \mathrm{C}$, just around the glass transition of $98^{\circ} \mathrm{C}$ (measured by DSC). As can be seen in Figure 11, folds have formed, but they have rather smooth curved shape, than sharp hinges. In first approximation they can be regarded as undulations of sinusoidal form, supporting herewith the theory of undulation instability. Further, if the transformation of the initial sinusoidal folds goes trough concentric-like to chevron folds, 
vestiges of the earlier concentric-like pattern should also be observable. We believe to detect them also in this sPS sample, on the right side of Figure 11.

On the other hand, the observed undulations are not very regular and less coherent in wavelength and amplitude, and in these features remind the pictures found in SBS block copolymers at strains below $20 \%$ [15]. In the cited work these observations are discussed as indications of the alternative mechanism of folding, assuming first kink nucleation at the defects and evolution to chevron morphology through further propagation of the kink boundaries into adjacent regions.

Obviously, our current observations are not sufficient for a definitive statement about the relative importance of these two mechanisms in semicrystalline polymers. It could be that both mechanisms are possible, but which will take place depends on the specific microstructure of the semicrystalline polymer.

In the regions where the chevron shape is well formed, the tilt angle can be evaluated. Near the $\mathrm{T}_{\mathrm{g}}$ it is relatively large, about $70^{\circ}$. With increasing deformation temperature the chevrons rapidly become sharper, and at only $10^{\circ} \mathrm{C}$ above the glass transition of sPS the average tilt angle amounts already $55^{\circ}$. The folds have become regular and coherent in wavelength, as depicted in Figures 6.

A comparison of the chevron patterns in different polymers shows a great variety of tilt angles: for $\beta$-iPP upon deformation at room temperature the typical tilt angle is $36-40^{\circ}$, in HDPE the limbs close to an average angle of $30^{\circ}$. In general, a trend of decreasing tilt angles with increasing difference $\Delta T$ between deformation temperature and glass transition is observed. The difference $\Delta T=T_{\text {def. }}-T_{g}$ accounts indirectly for compliance, mobility and viscosity of the soft phase, all of them being factors which affect the formation of chevron morphology under force. However the plot of the tilt angle in the different polymers as a function of $\Delta T$ is not a straight line. This result can be related to the complex influence of the microstructural details of each polymer on the chevron formation.

Another point is the dependence of tilt angle on deformation strain. For other layered systems it is shown that with increasing strain the chevrons become sharper, i.e. the tilt angle $\varphi$ between lamellae and deformation direction decreases. For a globally oriented lamellar SBS block copolymer a model of affine tilting of lamellae with macroscopic elongation $\lambda$ upon perpendicular deformation at room temperature was proposed [12]. Many years earlier a linear dependence of the tilt angle on the extent of roll in POM has been reported [17]. The situation in unoriented materials is expected to be more complicated. It should be mentioned however, that 
we observed nearly linear decrease of the tilt angle in the folded domains of unoriented sPS with increasing deformation. Detailed investigations in other polymers are still under way.

\section{§ 4. CONCLUSIONS}

(1) In this work we have demonstrated that chevron morphology can be formed under dilative strain not only in special polymeric materials with layered structure, but also in traditional semicrystalline polymers. The prerequisites therefore are layered lamellar arrangement and large difference in stiffness between the crystalline and amorphous phases.

(2) We showed that chevron folding is a common deformation mode which can appear in real, i.e. globally unoriented or partially oriented polymers, in areas where the lamellar stacks are oriented perpendicular to the deformation direction. Consequently, chevron folding in semicrystalline polymers has to be regarded as a possible microdeformation of equal importance as the three main modes generally accepted.

(3) The mechanism of chevron folding in semicrystalline polymers and the main characteristics of the chevron pattern are similar to that in other layered systems. The wavelength of the folding is affected by the surrounding lamellar domains. Accompanying details as simultaneous void formation, asymmetry etc. are influenced by the specific microstructure of each polymer.

\section{ACKNOWLEDGEMENTS}

The authors are indebted to Ing. S.Goerlitz and Ing. I.Naumann for a significant part of the TEM investigations presented in this work. 


\section{REFERENCES}

1. JOHNSON, A.M., 1977 Styles of Folding, (Elsevier, New York)

2. RAMSAY, J.G. and HUBER, M.I., 1987, The Techniques of Modern Structural Geology (Academic, London), Vol. 2;

3. MOLHO, P., PORTESEIL J.L., SOUCHE Y., GOUZERH J. and LEVY J.C.S., 1987, J.Appl.Phys. 61, 4188

4. SEUL, M. and WOLFE, R., 1992, Phys. Rev. Lett. 68, 2460.

5. SINGER, S.J., 1993, Phys. Rev. E 48, 2796

6. RIBOTTA, R., MEYER, R.B. and DURAND, G., 1976, J. Phys. (Paris), 35, L161.

7. RIEKER, T.P., CLARKE, N.A., SMITH, G.S., PARMAR, D.S., SIROTA, E.B. and SAFINYA, C.R., 1987, Phys. Rev. Lett. 59, 2658.

8. SHAlAGINOV, A.N., HAZELWOOD, L.D. and SLUCKIN, T.J., 1998, Phys. Rev. E 58, 7455

9. FUJIMURA, M.; HASHIMOTO, T. and KAWAI, H., 1978, Rubber Chem.Technol. 51, 215.

10. SÉGUÉLA, R. and PRUD’HOMME, J., 1981, Macromolecules, 14, 197.

11. HONEKER, C.C. and THOMAS, E.L., 1996, Chem. Mater. 8, 1702

12. COHEN, Y., ALBALAK, R.J., DAIR, B.J., CAPEL, M.S. and THOMAS, E.L., 2000, Macromolecules, 33, 6502.

13. YAMAOKA, I. and KIMURA, M. 1993, Polymer, 34, 4399.

14. HUY, T.A., ADHIKARI, R. and MICHLER, G.H., 2003, Polymer 44, 1247

15. COHEN, Y., BRINKMANN, M. AND THOMAS, E.L., 2001, J. Chem. Phys., 114, 984

16. READ; D.J., DUCKETT R.A., SWEENEY, J. and MCLEISH, T.C.B., 1999, J.Phys.D: Appl.Phys., 32, 2087.

17. GEZOVICH, D.M. and GEIL, P.H., 1971, J.Mat.Sci., 6, 509

18. GEZOVICH, D.M. and GEIL, P. H., 1971, J.Mat.Sci., 6, 531

19. SONG, H.H., ARGON, A.S. and COHEN, R.E., 1990, Macromolecules, 23, 870.

20. BARTCZAK, Z., ARGON, A.S., and COHEN, R.E., 1992, Macromolecules, 25, 5036.

21. WADE ADAMS, W., YANG, D. and THOMAS, E.L., 1986, J.Mat.Sci., 21, 2239

22. HOFMANN, D, GEISS, D, JANKE, A., MICHLER, G.H. and FIEDLER, P., 1990, J.Appl. Polym.Sci., 39, 1595

23. MICHLER, G.H., MARINOW, S. and NAUMANN, I., 1989, Plaste und Kautschuk, 36, 432. 24. MiCHLER, G.H. and MORAWIETZ, K., 1991, Acta Polymerica, 42, 620.

25. MICHLER, G.H., 1992, Coll. Polym. Sci., 270, 627 
26. LI, J.X., CHEUNG, W.L. and CHAN, C.M., 1999, Polymer, 40, 3641

27. MICHLER, G.H., ADHIKARI R. and HENNING S., 2004, J.Mat.Sci., 39, 3281

28. HENNING, S., ADHIKARI, R., MICHLER, G.H., BALTÁ CALLEJA, F.J. and KARGERKOCSIS, J., 2004, Macromol. Symp., 214, 157

29. Henning, S, MiChler, G.H., ANIA, F. and BAltá CALleJA, F.J., Coll. Polym. Sci., published online 1 December 2004

30 MICHLER, G.H., 1992, Kunststoff-Mikromechanik (Hanser Verlag, München, 1992)

31. LIU T., PETERMANN J., HE, CH., LIU, Z. and CHUNG T.S., 2001, Macromolecules, 34, 4305

32. EDWARDS, B.C. and PHILLIPS, P.J., 1974, Polymer, 15, 351

33. CRETON, C., KRAMER, E.J. AND HADJIIONNAU, G., 1992, Coll.Polym.Sci., 270, 399

34. GALESKI, A., 2003, Prog. Polym. Sci. 28, 1643

35. KAUSCH, H.H., GENSLER, R., GREIN, CH., PLUMMER, C.J.G. and SCARAMUZZINO, P., 1999 J.Macromol.Sci.-Phys. B38, 803

36. BAYLY, M.B., 1974, Tectonophysics, 24, 291.

37. BOWDEN, P.B. and YOUNG, R.J., 1971, Natur, 229, 23.

38. WANG, Z.G., 1994, J. Chem. Phys. 100, 2298

39. KAWAI, H. and HASHIMOTO, T. 1980, J. Macromol. Sci., B17, 427.

40. KRUMOVA, M., SEYDEWITZ, V. and MICHLER, G.H., 2002, Int. Conf. Polymer materials P2002, Halle (Saale), Sept. 2002

41. KAUFMANN, W.E. and SCHULTZ, J.M., 1973, J.Mat.Sci., 8, 41

42. PETERMANN, J. and SCHULTZ, J.M., 1978, J. Mat. Sci., 13, 50

43. HISS, R., HOBEIKA, S., LYNN, C. and STROBL, G. 1999, Macromolecules, 32, 4390

44. FIEDLER, P., MICHLER, G.H. and BRAUN, D., 1986, Acta Polym. 37, 241

45. TIMOSHENKO; S, 1988, Theory of elastic stability (McGraw Hill, New York)

46. HU, Y., HILTNER A. and BAER, E., 2004, Polym. Comp., 25, 653

47. RAMBERG, H., 1963, Bull .Am .Assoc. Pet. Geol., 47, 484

48. PATERSON, M.S. and WEISS, L.E., 1966, Geol. Soc. Am. Bull., 77, 343 


\section{FIGURE CAPTIONS}

Figure 1. Morphology of iPP: a) layered lamella arrangement in $\beta$-iPP; b) "cross-hatched" arrangement of the crystalline lamellae in $\alpha$-iPP (selectively stained ultrathin sections, TEM)

Figure 2. Chevron morphology in deformed $\beta$-iPP, deformation direction horizontal.

Figure 3. Disordered lamellar arrangement in LDPE (selectively stained ultrathin sections, TEM)

Figure 4. Lamella folding in HDPE-blow foils upon additional drawing, deformation direction vertical

Figure 5. Morphology of syndiotactic polystyrene (sPS), (selectively stained ultrathin sections, TEM)

Figure 6. Chevron structure in $\mathrm{SPS}$ after tensile deformation at $110^{\circ} \mathrm{C}$, deformation direction shown by the arrow

Figure 7. Schematic representation of the morphology of globally unoriented semicrystalline polymer: before deformation (top) and after deformation (bottom)

Figure 8. Coexistence of chevron pattern and microvoids in deformed samples of $\beta$-iPP, deformation direction horizontal

Figure 9. Chevron folds with different wavelengths in a sample of sPS, deformation direction shown by the arrow

Figure 10. Chevron pattern in deformed SBS block copolymer with lamellar morphology, deformation direction shown by the arrow

Figure 11. Morphology of sPS formed upon deformation at temperature around the glass transition, deformation direction shown by the arrow 


\title{
Chevron morphology in deformed semicrystalline polymers
}

\author{
M. KRumova*, S. Henning, G. H. MichleR \\ Martin-Luther-University Halle-Wittenberg \\ D-06099 Halle (Saale), Germany
}

\begin{abstract}
Chevron morphology was observed using transmission electron microscopy in various semicrystalline polymers deformed in tensile experiments. The morphological and mechanical prerequisites for chevron structure formation in semicrystalline polymers were revealed. It was demonstrated that chevron folding is a common deformation mode which can appear in real, i.e. globally unoriented or partially oriented samples, in areas where the lamellar stacks are oriented perpendicular to the deformation direction. Similarities with the behaviour of other layered systems were found. The mechanisms of chevron formation is discussed in the light of the fundamental statements of the folding theories and is related to the specific microstructure of the polymers. The effect of the boundary conditions, deformation temperature and macroscopic strain on the characteristics of the chevron structure is described.
\end{abstract}

Keywords: chevron, folds, semicrystalline polymer, deformation, morphology, electron microscopy

1

*Author for correspondence. E-mail: marina.krumova@iw.uni-halle.de http://mc.manuscriptcentral.com/pm-pml 


\section{§ 1. INTRODUCTION}

Folded structures can be often observed in layered materials at a great diversity of length scales. The earliest investigations on this phenomenon date back over a century and were performed in the field of the structural geology. Natural folds in rocks were studied in details and different types (sinusoidal, concentric-like, chevron, kink and asymmetric folds) were distinguished and classified. On the bases of these observations and experiments with model systems, theories about the mechanisms and mechanics of folding have been developed. There is an extensive body of literature devoted to the practical experience and theoretical treatments of layer folding. Some geology textbooks, e.g. [1,2] offer a detailed review of the most important results

Folds comprising straight limbs and sharp hinges are known as chevrons. Chevron patterns can be found in metamorphic, volcanic, and sedimentary terrains in many parts of the Earth. They range widely in scale, with wavelengths from a few $\mathrm{mm}$ to about $1 \mathrm{~km} \mathrm{[1].}$

Formation of chevron morphology at a scale of about $100 \mu \mathrm{m}$ has been observed later in thin magnetic films, comprising stripe phases as a pattern of self-organisation. When the stripe phases are strained by changing either the temperature or the applied magnetic field in a direction tending to decrease the equilibrium stripe spacing, the stripes buckle in order to bring the local layer spacing closer to equilibrium [3,4]. The initial modulation appears above a certain strain threshold and is sinusoidal. With increasing strain the stripe modulation pattern incorporates higher Fourier modes and takes a zigzag or chevron appearance [5].

Chevron folding occurs even at molecular length scale - in liquid crystals confined between the flat parallel boundaries of the solid cell. The buckling phenomena has been observed in the homeotropic geometry, when the smectic layers are parallel to the plates [6], as well as when they are perpendicular to them, in "bookshelf" geometry [7]. The chevron is believed to be a consequence of the mismatch between the natural thermodynamically stable smectic layer thickness and that imposed by layer pinning at the cell surface [8]. The undulation is generated either by explicit tension perpendicular to the layers or simply by changing the temperature. The wavelength is imposed by the cell size and usually amounts some micrometers.

In the mentioned layered systems folding occurs as a response to deformation force acting perpendicular to the layering, The phenomenon seems to be based on the same fundamental mechanisms in very different materials, on very different length scales ranging from macroscopic geological formations to the molecules of smectic liquid crystals. 
Chevron pattern attracted again scientific attention with the study of the deformation behaviour of thermoplastic elastomers, such as styrene-isoprene-styrene or styrene-butadienestyrene copolymers. It was found that if SIS or SBS copolymers in a globally aligned hexagonal or lamellar phase are stretched perpendicular to the rods or plates, then above a critical strain the lamellae or cylinders buckle to form a chevron or zig-zag structure. The morphology was detected and characterised by different investigation techniques, SAXS [9, 10], combined SAXS measurements and transmission electron microscopic (TEM) observation [11, 12], TEM [13]; Fourier transform infrared (FTIR) spectroscopy [14] and Scanning force microscopy (SFM) [15]. In a recent work [16] Read et al. modeled the instability towards chevron folding in layered block copolymers in terms of the macroscopic elastic moduli of the material, making corrections to account for microscopic deformations caused by the buckling.

The morphology of the majority semicrystalline polymers exhibits alternating crystalline lamellae and interlamellar amorphous spaces and thus approaches a nanoscaled layered system, even if with lower perfection. Consequently, upon loading perpendicularly to the lamellar stacks, formation of chevron structure can be expected. Actually, chevron structure has been observed in some polymers as a result of special processing, in the aim to produce single crystal-like texture. In the 1970s, various polymers have been subjected to cold-rolling and the structural changes which occur during rolling have been studied in detail. Chevron structures have been observed by TEM in polyoximethylene (POM) [17] and polyethyleneoxide (PEO) [18], and called "banded zig-zag type of lamellar structure with the bands parallel to the roll direction". Later, chevron like morphology has been found in high density polyethylene (HDPE), again as a result of special processing, rolling and channel-die compression, e.g. [19, 20], melt-drawing [21] or film blowing, e.g. [22]. All these investigations were predominantly concerned with lamella tilting as a result of the processing, the strange "zig-zag" morphology was not attributed to a special deformation mode. Analogy with similar structures in other systems was not realised.

Only few works describe the formation of this morphology in traditional tensile experiments. The materials studied were HDPE and UHMWPE [22-25]. However, neither in these publications the term "chevron" was used, the morphology was either mentioned without any denomination or was called "herring-bone" or "fir tree-like".

Recently a study focused on the mechanism of polymorphic transformation of $\beta$-PP into $\alpha$-PP upon deformation has been published [26]. On some of the TEM micrographs, presented in the publication, chevron morphology can be easily recognised. The authors, however, mention this behaviour as shearing and tilting of broken horizontal lamellae towards the loading direction. 
In the last years we repeatedly found chevron pattern in other polymers deformed in traditional tensile experiments. Our observations for polypropylene we presented in recently appeared publications [27-29].

In this report we are not going to present the result of one particular investigation, rather we wish to summarise the material accumulated over the past years in our laboratory. Further in this work we aim:

(1) to extend and generalise the experience on this phenomenon in semicrystalline polymers

(2) to demonstrate that chevron folding is a common deformation mode which can appear in a real, i.e. globally unoriented or partially oriented semicrystalline polymer in regions where favourable conditions exist

(3) to compare and correlate the main characteristics of chevron pattern formation in semicrystalline polymers with the existing theories on mechanism and mechanics of folding in other layered systems.

\section{$\S 2$. EXPERIMENTAL DETAILS}

\subsection{Materials}

For this investigation we selected materials, which represent different classes of semicrystalline polymers.

Polypropylene (PP) was chosen as a material from the family of commodity plastics. Samples were prepared from high molecular weight commercial isotactic polypropylene grades (Borealis AG): $\alpha$-iPP was Daplen BE50 and $\beta$-iPP was Daplen BE60. Sheets with thickness of 1 $\mathrm{mm}$ were produced by compression moulding and subsequent multistage crystallisation procedure in the second $\beta$-iPP case.

Syndiotactic polystyrene (sPS) is a relatively new material and was interesting for our study due to its high glass transition temperature, so that deformation at common conditions (room temperature) practically takes place with amorphous phase in glassy state. The used sPS was commercial grade (Dow Chemical Corp.) with molecular weight $220000 \mathrm{~g} / \mathrm{mol}$ and syndiotacticity of $98 \%$. Plates of $1 \mathrm{~mm}$ thickness were prepared by compression moulding and crystallisation at $245^{\circ} \mathrm{C}$ to obtain the $\alpha$-sPS modification and by slow cooling to obtain the $\beta$-sPS modification. We report here for the first time about formation of chevron morphology in sPS upon deformation. 
In addition we used results of previous investigations of HDPE and UHMWPE, where chevron formation was first found in our tensile experiments. Some of these results are reviewed in the book on micromechanics in polymers by Michler [30].

\subsection{Experimental Techniques}

\section{Mechanical Testing}

Macroscopic tensile deformation experiments were carried out using universal tensile machine Zwick 1425 (Ulm, Germany) and a MINIMAT miniature materials testing device (Polymer Laboratories, UK). Standard tensile bars were prepared from the pressed sheets for the Zwick machine. For the MINIMAT device miniaturised, dumbbell-shaped tensile bars were punched out of the plates using a special pierce tool. Tensile deformation experiments were performed at a cross-head speed of $1 \mathrm{~mm} / \mathrm{min}$ at various temperatures. The strain rates in both cases were of the order of $2.10^{-4} \mathrm{~s}^{-1}$.

\section{Transmission electron microscopy (TEM)}

Ultrathin sections of about $70 \mathrm{~nm}$ were prepared from regions close to the middle of the tensile bars, corresponding to different states of elongation. The sections were cut parallel to the tensile direction by means of a Leica Ultracut microtome equipped with a Diatome diamond knife. For contrast enhancement the materials were stained with $\mathrm{RuO}_{4}$ vapour. Micrographs were acquired using a JEOL transmission electron microscope JEM 2010, operated at $200 \mathrm{kV}$ accelerating voltage. On the micrographs the crystalline lamellae appear bright and the amorphous regions dark.

\section{$\S 3$. RESULTS AND DISCUSSION}

\subsection{Prerequisites for chevron pattern formation in semicrystalline polymers}

On the bases of a great amount of results obtained by direct imaging of microdeformation structures in various polymers, we can conclude that not in each polymer chevron pattern are formed upon deformation. Some prerequisites that enable the chevron folding must be present. 


\section{Morphology}

As a first prerequisite we consider the morphological one, that is the existence of stacks of nearly parallel crystalline lamellae, i.e. layered arrangement, as for example, in the case of the $\beta$-polymorphic modification of isotactic polypropylene ( $\beta$-iPP), shown on Figure 1a. The lamellae in this arrangement are able to undergo cooperative folding (Figure 2) when dilative force is applied normal to the layers. A great difference exists between this morphology and that of the more common $\alpha$ polymorphic modification of iPP, as evident from Figure $1 b$. Characteristic for $\alpha$-iPP is the so called "cross-hatched" arrangement of the crystalline lamellae. The main lamellae ("parent lamellae") are growing radially from an initial site (centre of the spherulite), and "daughter lamellae" are formed by an epitaxial growth onto them at an angle of about $81^{\circ}$. In this way a kind of network is formed, not prone to chevron folding during deformation.

Morphological differences present also high (HDPE) and low (LDPE) density polyethylene. The disordered lamellar arrangement of LDPE (Figure 3) does not favour chevron formation upon deformation. In contrast, HDPE forms stacks of parallel lamellae, which undergo rotation and folding when perpendicular force is applied. This behaviour has been shown in [23] and [25] for HDPE-blow foils upon additional drawing (Figure 4). Similar behaviour has been reported also for ultra high molecular weight polyethylene UHMWPE after straining [24].

Analogical situation offers the comparison of isotactic (iPS) and syndiotactic (sPS) polystyrene. In iPS the lamellae are curved as a result of the lamellar stacks growth outward from the central regions of the spherulites by splaying and branching [31, 32] Thus, the stack can hardly be regarded as a nanolayered system of nearly parallel layers. In contrast, the lamellar arrangement of sPS shown on Figure 5, looks very similar to that of $\beta$-iPP, represented on Figure 1a.

Nevertheless sPS does not form chevron patterns when deformed at room temperature, this means that the morphological prerequisite is not sufficient for chevron formation.

\section{Alternating hard and soft layers}

The concept of alternating hard and soft layers emerges in the earliest investigations on folding. It has been realised that for fold formation slippage between layers and flowage of material from limbs toward crests and troughs is needed, i.e. angular folds can develop when the layers have markedly different physical properties. Layers of different mechanical compliances show different resistance to the applied deformation stress, they react and adjust their strain by 
buckling at a point of instability. The soft layers play an important role: they start to deform plastically and ensure the strain needed for initiation of the bending of the hard layers.

In polymer physics the condition of alternating hard and soft layers is well illustrated with the deformation behaviour of block copolymers with lamellar morphology. Styreneisoprene-styrene and styrene-butadiene-styrene copolymers comprise alternating glassy polystyrene and rubbery butadiene or isoprene lamellae and form chevrons upon deformation at room temperature. Differently, in styrene - methyl methacrylate block copolymers, with lamellar morphology, both phases are hard (in glassy state) at room temperature. Stretching provokes in this case crazing and not chevron folding [33].

The studied semicrystalline polymers (HDPE and $\beta$-iPP) can be regarded as layered systems of hard crystalline lamellae and soft amorphous interlamellar layers. When deformation is applied at room temperature, the amorphous phases of these polymers are really soft, since being well above their glass transition. The same holds also for the above mentioned POM and PEO, in which chevron structure has been observed as a result of cold rolling (see $\S 1$. Introduction). Due to their very low glass transition temperatures of $-83^{\circ} \mathrm{C}$ and $-67^{\circ} \mathrm{C}$, respectively, the amorphous interlamellar regions are soft when deformation is performed.

Differently behaves a semicrystalline polymer with glass transition above room temperature. The syndiotactic polystyrene (sPS) we chose as a model for such polymers, fulfils the morphological prerequisite (see Figure 5). But due to its high glass transition temperature of nearly $100^{\circ} \mathrm{C}$, the amorphous layers are far from being rubbery, the mobility is restricted and the material deforms at room temperature in a brittle manner. Only after raising the deformation temperature above $100^{\circ} \mathrm{C}$, the required mobility (rubberlike state) is reached and chevron pattern appear in corresponding areas, as demonstrated on Figure 6.

From these observations the second prerequisite can be deduced, i.e. a large difference in the mechanical and/or thermal properties of the crystalline and amorphous phases of the polymer.

The condition of difference in the physical properties of the two type of layers has been extensively discussed in the literature on folding. The difference between hard and soft is expressed in terms of stiffness, elastic modulus, compliance, mobility, viscosity, glass transition temperature etc., depending on the multilayer and on the model used for investigation of the phenomenon. In the geology usually viscosity difference is considered. It has been shown that even the fold shape depends on the viscosity ratio of the two types of layers [2]. In multilayers an additional factor is also important in controlling fold shape. It is found that, for any two materials of particular ductility contrast the curvature of the fold surfaces is strongly affected by the 
proportion of softer to stiffer material (the $n$-value). With a decrease in the proportion of softer material high curvatures are concentrated near the hinge zone and the fold limbs become less curved, so the overall shape approaches more closely the sharp-hinged, parallel-limbed chevron fold [2]. At moderate $n$-value, which is relevant for the case of semicrystalline polymers, the formation of chevron folds is favoured at high viscosity (ductility) contrast. The question about a "threshold contrast" is more complicated. It can be evaluated for each particular multilayer using the mathematical expressions for the initial wavelength of folding derived from the theoretical treatments. Some of these expressions will be discussed in more details in $\S 3.4$.

In polymer physics predominantly Young's-modulus difference of the two type of layers is considered. Finite element modelling of the folding process in styrene-butadiene block copolymers with lamellar morphology has followed the development of the fold shape at different E-modulus - ratios [16]. In a single layer chevron pattern is observed to form at an Eratio of 100 , in a multilayer, with the particular parameters selected, first sinusoidal folds develop at E-ratio of 500 between stiff and soft layers.

Generally, in styrene-butadiene block copolymers a large difference between the Young moduli of PS and PB exists, with a ratio up to 1000 times. In the case of semicrystalline polymers as a modulus of the amorphous phase the value of the rubbery plateau can be considered, which usually amounts some decades MPa. Then, the ratio between the moduli of crystalline and amorphous phases will be of the order of x1000 to x10 MPa.

\subsection{Mechanism of chevron folding in semicrystalline polymers}

A real sample of a semicrystalline polymer between the grips of the tensile stretching device is usually globally unoriented or only partially oriented, comprising lamella stacks randomly distributed in various directions. Schematically such material can be represented as consisting of regions (domains) of parallel lamellae, the domains themselves situated at different angles with respect to the deformation direction. (Figure 7a).

All three currently established local deformation modes can take place in the lamellar stacks in these domains. Whole stack (domain) can rotate if it is surrounded by amorphous matrix and hence, free to rotate under the stress. In the domains comprising lamella stacks at some angle to the stretching direction interlamellar shear is the most probable deformation mechanism. It involves slip of the lamellae parallel to each other with the amorphous phase undergoing shear, which is a relatively easy deformation mechanism for a polymer above the 
glass transition temperature [34]. With stretching, the stacking axes of the lamellae in these domains get closer to the stretching direction, this enables longitudinal extension together with lateral contraction of the domains.

The object of interest for this investigation are the domains comprising lamella stacks perpendicular to the stretching direction, as the central domain " $\mathrm{A}$ " in the scheme of Fig. 7a. According to the established local deformation modes this domain initially mostly deforms by interlamellar separation. As known, the amorphous regions are entangled networks solidly attached to the adjacent lamellae, the lamellae are hold together by tie molecules through the amorphous phase. At the beginning of macroscopic stretching, the lamellae are displaced rigidly, and the ensuing interlamellar separation leads to strong hydrostatic tension within the constrained amorphous network. The tie molecules become more extended and transfer locally concentrated elastic stresses to the lamellae [35]. Stress relief can occur in two ways, through cavitation within the amorphous network and/or through crystal plastic deformation.

At the same time this domain of parallel lamellae can be regarded as a multilayer subjected to a deformation force perpendicular to the layering. Additionally, a contraction stress component is acting parallel to the layering due to the plastic deformation of the neighbouring domains (macroscopically expressed as the Poisson ratio). Then, like other layered systems, after reaching a critical strain this lamella stack should undergo folding. Qualitatively this behaviour can be explained with the aim of the lamella stack to recover the equilibrium spacing and not to change significantly the volume, similar to the mechanism established for other layered systems (see $\S 1$. Introduction). According to theoretical treatments of deformation in multilayers, folds (angular or rounded) may result from the natural tendency of materials to deform in energy minimising way [36]. These theoretical treatments have further demonstrated that angular folds (chevrons, kinks etc.) have the most economic profile as regards energy consumption. Recently, Cohen et al. [15] demonstrated that also in SBS block copolymers with lamellar morphology bending of the hard layers is less costly in free energy than dilation of the layers, which results in folding of the layers by kinking or undulation into chevron morphology.

In semicrystalline polymers the situation may be more complicated. As mentioned above stress relief can occur in two ways, through cavitation within the amorphous network and/or through crystal plastic deformation. Due to the great number of defects (such as chain ends or weak entanglements) cavitation in the amorphous regions can easily occur. Under tensile stress both processes can be observed: i) chevron folding, which involves lamella plastic deformation and damps microvoid formation or/and ii) lamella separation with cavitation. One of these 
concurrent processes or both simultaneously will take place in dependence on the specific characteristics of the polymer.

It is established that plastic deformation of polymer crystals is generally crystallographic in nature, the main mechanism being crystallographic slip, twinning and martensitic transformation. The slip mechanism is the most important since it can produce larger plastic strains. Slip takes place when the resolved shear stress on the slip plane reaches a critical value, known as the critical resolved shear stress. Cavitation during deformation can be observed in polymers for which the critical resolved shear stress for the easiest slip system of crystals is higher than the stress required for cavitation [34]. In the other cases, crystallographic slip will occur earlier, relaxing the stress and a cavitational pore will not appear. Hence, it can be expected that in latter polymers chevron folding will be facilitated at low strains.

Our experiments on HDPE show only chevron folding and no cavitation upon straining perpendicular to the lamellae stacks. This behaviour can be explained with the relative low values of the resolved stress for the easiest slip (shift in chain direction) in HDPE, between 7.2 and 15.6 MPa [20, 37]. On the other hand, it has been shown that the values for the resolved stress depend also on the crystal thickness, so that cavitation can be found in HDPE slowly cooled or formed in thick wall products [34]. The HDPE investigated in our laboratory comprised relatively thin lamellae, of the order of $15 \mathrm{~nm}$. In HDPE samples with thicker lamellae [21] simultaneous kink formation and cavitation of the amorphous regions are observed upon tensile deformation.

In $\beta$-polypropylene chevron pattern and microvoids can be simultaneously observed in deformed samples, as illustrated in Figure 8. Consequently, both competing processes take place. According to Galeski [34] $\alpha$-polypropylene belongs to the polymers in which cavitation during deformation can be expected, due to its relative high value of the resolved stress for the easiest slip (in chain direction), around 22-25 MPa. Unfortunately, there are no data about this stress in $\beta$-PP available yet in the literature. Based on the facility for chevron folding in $\beta$-polypropylene we could suppose that the critical resolved stress for the easiest slip in $\beta$-PP is lower than in $\alpha$ PP and comparable with the critical stress for cavitation.

It should be mentioned that the critical resolved shear stresses for slips are well known only for few polymers. They were measured using samples of rather well defined texture. As expected this parameter is yet unknown for the relatively new syndiotactic polystyrene (sPS), which shows chevron morphology upon stretching (see Fig.6). The frequent appearance of 
chevrons and the absence of voiding in this polymer could be an indication of relatively low critical resolved shear stresses for slips in its lamellae.

Beside the resistance to plastic deformation of the crystal phase, the constraints on the amorphous phase have to be taken in account. In the vicinity of the glass transition a higher quantity of voids can coexist with chevron folds due to the reduced mobility and deformability of the amorphous network.

\subsection{Critical strain}

It is well established that the initial layered structure transforms to chevron morphology beyond a critical strain. Of practical importance would be a relation of this transformation to changes in the stress-strain behaviour. However, in our case of globally unoriented semicrystalline polymers this issue is difficult to be resolved. We can only estimate the local strain in the vicinity of the domain which forms chevrons and indicate the approximate region of the macroscopic stress-strain curve, where chevron formation can be expected. Some qualitative considerations for the upper strain limit can be deduces in addition from works on morphological changes upon deformation which are focused on the onset of fibril formation.

More attention is paid on this problem in block copolymers with nearly perfect lamellar arrangement of the hard and soft phases. Since they can be regarded as a model of nanolayered polymer, we shall make use of some results from these investigations. Among the published works two main views can be distinguished. Some authors associate the critical strain with the yield point and predict layer folding at very low strains of only some percent $[16,38]$. Others [e.g. 15] found that deformation perpendicular to the layers proceeds at room temperature uniformly without any noticeable necking and the four-point SAXS pattern, characteristic for chevron structure appears only beyond the yield point when the stress-plateau is reached. In other works on globally oriented SBS block copolymers values for the critical macroscopic strains of about 30 or $40 \%$ are reported [9, 13, 39]. Interesting for us is an investigation of globally unoriented SBS samples [10]. Chevron morphology formation was observed there at about $20 \%$ strain.

Our findings for unoriented semicrystalline polymers correlate well with the latter results. SEM and TEM micrographs of $\beta$-PP deformed at room temperature show formation of chevron pattern at about $20 \%$ local deformation. In the above cited work on polymorphic transition of $\beta$ PP upon deformation [26] a TEM micrograph shows chevron pattern at local strain of about 
$35 \%$. We suppose that chevron morphology has appeared at smaller deformation, but the corresponding pictures are not shown, because the attention in the study is focused on other structures.

In sPS we performed the deformation at higher temperature for the reasons explained in $\S$ 3.1. We observed formation of chevron morphology also at roughly $20 \%$ local strain. The corresponding stress-strain curves did not show any noticeable necking. [40].

We have not done specific investigations for measurement of the critical strain in HDPE beyond which chevron pattern appear. Nevertheless on the basis of works devoted to the general deformation behaviour of HDPE, some estimations can be made. In early studies, dating back over 30 years, it has been found that the critical strain level for texture developing in polyethylene upon deformation at room temperature appears to be near $20 \%$ [41]. Subsequent detailed investigation on the deformation behaviour in spherulitic PE by Petermann and Schultz [42] has shown that upon tensile straining at low deformation rates PE behaves reversibly for extensions up to $40 \%$. Higher elongation is needed for initiation of the other deformation modes which are mainly plastic, as slip, chain kinking etc. Other investigations dealing with the deformations in PE also associate the onset of plastic deformation modes with macroscopic elongation beyond $20 \%$. Analysing the transition points in the true stress-true strain curve Strobl et al. [43] associated the Hencky strain region between $\varepsilon_{\mathrm{H}} \approx 0.1$ and $\varepsilon_{\mathrm{H}} \approx 0.6$ (corresponding to nominal strains between 10 and 80\%) in PE with collective slip processes. They also suggested that the "coarse" block slip processes should be dominant. Up to $\varepsilon_{\mathrm{H}} \approx 0.6$ the lamellae, although set together of displaced blocks, still exist. If we make use of this schema, we can expect chevron folding to occur beyond $10 \%$ and begin to disappear beyond $80 \%$ nominal strain.

It is interesting to remind here some observations from cold rolling of polymers. Rolling is similar to drawing in that the tangential (frictional) force of the roller provides a component which exerts a tensile stress on the material. Therefore, rolling can be considered to be a combination of drawing along the roll direction and compression along the thickness direction. In rolled POM [17] chevron like morphology has been observed at extend of the roll $\mathrm{L} / \mathrm{L}_{0}=0.8$ (i.e. deformation of about $20 \%$ ).

Summarising all these results, it appears that chevron formation in semicrystalline polymers can be associated with the region of the stress-strain curve beyond the yield point, when the stress-plateau is reached. At the low strain rates used in these experiments, the critical strain varies with the material roughly between 15 and $40 \%$.

From microstructural point of view, the crystalline lamellae start to deform plastically (slip is initiated) after the entangled non-crystalline chains have reached their elongational limit. 


\subsection{Characteristics of the chevron morphology and correlations}

In the literature dealing with chevron morphology the following main characteristics are discussed: wavelength, layer spacing and the tilt angle of the layers with respect to the stretching force. In the following we will make an overview of the chevron structures observed in the studied polymers and describe their changes with deformation temperature and strain.

\section{Wavelength}

From the evaluation of the great amount of micrographs showing chevron morphology in the studied semicrystalline polymers we can conclude that the typical size of the regions (domains) undergoing chevron folding lies between 1 and $2 \mu \mathrm{m}$ and that in average 3-4 folds are accommodated in these regions. The wavelength varies between 200 and $500 \mathrm{~nm}$ with material, strain and deformation temperature. Often folds with different wavelengths can be found in one sample, as the enclosed by the circles in Fig. 9. The chevron folds are not always symmetrical, 
often one arm ("limb") is longer than the other. The micrographs show further that symmetrical and asymmetrical folds can coexist in one sample, in different domains. In the case of $\beta$-PP, for example, this can be seen comparing Fig. 2 and Fig. 8.

All these peculiarities can be explained using the results of the general theories of folding developed for other layered materials. Now the mechanism of undulation instability is prevalently accepted for the response of layered materials to deformation perpendicular to the layers. According to it above a critical stress the multilayered materials buckle, with an initially sinusoidal buckling profile.

The undulation instability is a highly cooperative phenomenon of layers between confining external boundaries. The boundary conditions force adjacent layers to maintain their parallel orientation and limit the ability to undulate with a long wavelength. In this way the sample distance between the imposed boundary conditions is important in determining the wavelength of the buckling. The stable wavelength in the multilayered stack is affected further by the average properties of the material of the boundary (or outside of the stack). In the case of liquid crystals the boundaries are the parallel flat walls of the cell. In a perfectly aligned layered polymer the correlation distance for which the layers are coherently packed, could be in first approximation the macroscopic distance between the grips of the tensile stretching device. However, it is in practice very difficult to obtain in polymers such an ideal macroscopic arrangement. As mentioned in $\S 3.2$. a real sample, globally unoriented or partially oriented, can be schematically represented as consisting of domains of parallel lamellae, the domains themselves situated at different angles with respect to the direction of deformation (Figure 7). Chevron folding is completed only in domains with lamellar orientation perpendicular to the applied force. Consequently, the boundary conditions in this case will be imposed by the surrounding domains. Evidently, the boundary conditions are different for each domain which undergoes chevron folding. Moreover, the boundary conditions also change in the process of deformation.

The wavelength of the chevron profile is sensitive to both, to the boundary conditions on the multilayered material, and to the details of its structure. Mathematical expressions for the dominant wavelength of the folds which can develop in a system under external stress have been derived from the theoretical descriptions of the origin of folding. Most of the theoretical research has been devoted however to understanding of folding of single layers embedded in a surrounding medium, so that the theory of single-layer folding is quite highly developed. In the case of geological folding (a general solution for viscoelastic materials) the initial fold 
wavelength is shown to be controlled by layer thickness and viscosity contrast $[1,2]$. The following equation for the initial (sinusoidal) wavelength $\lambda$ is currently accepted:

$$
\lambda=2 \pi d\left(\sqrt[3]{\mu_{h} / 6 \mu_{s}}\right)
$$

where $\mu_{h}$ and $\mu_{s}$ are the viscosities of the hard and soft layers respectively, and $d$ is the thickness of the hard layer.

A linear dependence of the critical (initial) wavelength on the layer thickness has been derived also in the engineering mechanics for buckling of a single beam (layer) supported by elastic foundation [45]. Recently the linear dependence has been proved experimentally also for a 3-layer polymer system (elastomer-plastic-elastomer films) [46]. The following relationship involving the elastic moduli $E_{P}$ and $E_{R}$ of the plastic and rubbery layers has been proposed, which is more practical for calculations in the case of semicrystalline polymers than (1):

$$
\lambda=\pi d\left(\sqrt[3]{4 E_{P} / 3 E_{R}}\right)
$$

In multilayers these relationships are getting more complicated due to the great variety of possible combinations of material properties, layer thicknesses and interrelations between the two types of layers. In the simplest multilayer model of an infinite stack of regularly alternating layers the development of folds is controlled also by the viscosity contrast, but instead of the thickness of the stiffer layer, by the proportion $n$ of softer to stiffer layer thickness [2]. When $n=$ $\infty$ the solution coincides with the well defined initial wavelength developed in a buckled single stiff layer in softer medium deduced from equation (1). As the stiffer layers come to lie closer together (decreasing $n$-value), for a given stiff layer thickness, the characteristic initial wavelength becomes larger.

The features of the folded structure we observed in the studied semicrystalline polymers are in good agreement with the above model. An approximated calculation of the wavelength $\lambda$ of a buckled single lamellae according to equation (2), gives for typical lamella thicknesses in the range $10-15 \mathrm{~nm}$ values for $\lambda$ between 250 and $400 \mathrm{~nm}$. In the folded lamella stacks we always measured larger wavelengths for the respective polymers.

The exact mathematical expressions for the initial wavelength of folding multilayers, even composed of identical stiff layers and identical soft layers are very complicated. The wavelength defined according to the elementary theory of folding (the Ramberg wavelength) [1]

$$
\lambda_{R}=2 T \sqrt{\pi m / N} \sqrt[4]{\left(B_{1} / 12 B_{v}^{\prime}\right) /(1-D)}
$$


includes in addition dependence on the total thickness $T$ of the multilayer and the number of pairs of layers $N . B_{v}$ and $B_{v}{ }^{\prime}$ are calculated in terms of the layer thicknesses $t_{i}$ and moduli $B_{i}$ according to:

$$
\begin{aligned}
& B_{v}=\left(t_{1}+t_{2}\right) B_{1} B_{2} /\left(B_{1} t_{2}+B_{2} t_{1}\right) \\
& B_{v}^{\prime}=B_{v}\left(1+t_{2} / t_{1}\right)^{3}
\end{aligned}
$$

Here $i=1$ refers to stiff and $i=2$ to soft layers and:

$$
B_{i}=E_{i} /\left(1-v_{i}^{2}\right)
$$

where $E$ is Young's modulus and $v$ is Poisson's ratio.

The factors $m$ and $D$ are interrelated in a complex manner and must be determined iteratively. Graphical solutions to these equations for various thickness and moduli ratios can be found in the literature. We used them for an approximate estimation of the expected initial wavelength of the folds in our semicrystalline polymers.

According to the TEM micrographs the thickness ratio of amorphous layers to crystalline lamellae vary between 1 and 2 . For modulus ratio $B_{2} / B_{1}$ we adopted the approximate value of 0.01 and calculated $B_{v}$ and $B_{v}{ }^{\prime}$ after eq. (4) and (5). As mentioned above the typical size of the domains we found to undergo folding in our polymers lies between 1 and $2 \mu \mathrm{m}$, this corresponds to the total thickness $T$ of our multilayer; and the number of pairs of layers $N$ is the ratio of $T$ to the long spacing. Thus $N$ varies in the studied polymers in the range from about 60 to 200. Using these values we determined the ratio $\lambda / T$ from the graphical solutions for (3), presented in Fig.4, p.385 of [1]. We obtained results of $0.2-0.3$ in average, which are in a very good agreement with our experimental observations.

It should be mentioned that equations (1) and (3) describe the values of the wavelengths of the initially formed folds, which have sinusoidal form. Nevertheless, the use of these equations for the wavelength of chevron folds is also justified. It is established that with increasing strain the undulations evolve into chevron shape. The linearized theory of folding, proposed by Johnson [1], describes mathematically this transformation of the initial sinusoidal folds trough concentric-like to chevron folds and shows that the wavelength is related to the dimensional and rheological factors approximately as shown in eqs. $(3-6)$. The sinusoidal pattern becomes modified by high order effects, but the wavelengths of the final chevron folds, measured along layers, is roughly equal to the wavelength of the initial sinusoidal folds.

In the globally unoriented polymer samples domains (stacks) of different sizes and correspondingly different numbers of layers can be found. From the above relations it can be deduced that folds can form simultaneously in several domains, if they contain the same number 
of layers with identical elastic properties. But the wavelengths would be different if the thickness of individual layers were different in the separate multilayers. The wavelengths can be different also when individual layer thickness is the same, but the number of layers in separate domains differs. In semicrystalline polymers these structure details are the degree of crystallinity, lamella thickness and elastic characteristics of the crystalline and amorphous phase of each polymer. Obviously, in a real sample variations in lamella thickness and length always exist, as well as in number of layers in separate domains. Thus, folds with different wavelengths can be formed in one semicrystalline sample.

The coexistence of folds with different wavelengths, as illustrated in Fig. 9, can be also explained as a consequence of the equal probability for fold formation with different wavelengths. According to the theoretical modelling, with closing together of the stiffer layers (decreasing $n$ ), initial fold wavelengths over a wide range of values are equally favoured and the initial wavelength in the multilayer can become very irregular [47]. For the semicrystalline polymers studied, with their moderate to low value of $n$, typically about 1 , these results could be valid. At some conditions the folds which are most stable have the largest wavelength possible in the system, probably governed by the mechanical imperfections in the multilayer. From aspect of the semicrystalline microstructure these imperfections are related to local variations of the degree of crystallinity and lamella thickness, to defects in the crystalline structure and variations in density and length of the tie molecules connecting adjacent lamellae.

On the other hand chevron pattern with similar wavelength and amplitudes can be observed on the micrographs, although the number of lamellae and their thickness are not equal in all domains. This fact can also find its explanation in the graphical solutions to the equations of folding. According to them, appropriate combinations of the dimensional and rheological properties can result in similar wavelengths for very different multilayers [1].

An important peculiarity of the chevron morphology in semicrystalline polymers is the asymmetry of the folds. Asymmetry often exists also in geological formations. There it is explained as a result of interactions of folds with different wavelengths in the process of formation. The probability of such mechanism can be associated with the inhomogeneous initial structure of the semicrystalline polymers. In a real sample a perfect alignment of the lamellae parallel to each other is impossible. The lamellae are growing usually radial from an initial site (centre), so that certain curvature is inevitable. Further, in a real sample a distribution in lamellar thickness always exists, as well as different kind of defects. It may well be that these variations, lamellar waviness, dislocations and defects provide another length scale of folding in some regions. In the preceding paragraph the important role of the tie molecules for initiation of the 
lamella plastic deformation was pointed out. In a real sample obviously a density and length distribution of the tie molecules exists and this inhomogeneity can additionally affect the local folding.

In this way the asymmetry can be understood still accepting that chevrons are formed by undulation instability. On the other hand, the distortions of the chevron shape can be also explained if an alternative mechanism of chevron formation is supposed. Theoretical treatments and geometrical analysis have demonstrated that chevron folds can result from interaction of conjugate kink folds [48]. Recently, Cohen et al [12,15] assumed in their investigations on deformation of SBS block copolymers that kink bands first nucleate around defects and the further propagation of the kink boundaries into adjacent regions leads to chevron morphology.

Such mechanism is conceivable for semicrystalline polymers due to the great amount of defects in their multilayers. Nevertheless, we did not find in the polymers studied any indication of this mechanism. At the deformation conditions used in our investigations we did not observe kink formation, neither coexistence of kink bands with chevron folds. It should be mentioned however, that we have not followed the deformation process "in situ". The observations in TEM are made after the deformation is completed, so that the final result of the deformation is imaged. If we assume that the chevron folding is initiated by kink nucleation, we should further suppose a very fast transformation of the kinks to chevrons in the whole volume of the domain of interest. Some more details about this problem are discussed below, in § Tilt angle.

\section{Layer spacing}

Even the earliest studies of chevron profiles in rocks found that harder beds (layers) maintain roughly constant thickness whereas the softer layers thicken in the hinges. The analytical treatments of the transition from sinusoidal undulations to chevron morphology applicable to layered geological structures [36] and to smectic liquid crystals [5] and striped magnetic patterns [4] also predict changed in the layer spacing with deformation.

For the investigation of this process in thermoplastic block copolymers and rolled semicrystalline polymers predominantly SAXS and electron microscopy were used. It was concluded that in SBS block copolymers with layered morphology the long spacing remains nearly constant in the straight limbs of the chevron folds, but in the hinge regions the soft PB layers are more dilated than the PS ones. Our observations on chevron morphology in deformed SBS block copolymers (Figure 10) also support this statement. 
The electron microscopy investigations we performed of the chevron structure in semicrystalline polymers show also some thickening of the dark layers (corresponding to the amorphous phase) in the hinge regions, see Figs. 2, 3, 6. Consequently, the general features of the chevron structure in layered systems are present also in the folded domains of deformed semicrystalline polymers. This thickening of the amorphous phase in the hinge regions can be better distinguished when the difference between test temperature $\mathrm{T}_{\text {def }}$ and glass transition temperature $\mathrm{T}_{\mathrm{g}}$ of the polymer is larger. Figure 4 illustrates the larger dilation in the hinge region in HDPE, compared with $\beta$-PP (Fig. 2) and sPS (Fig.6); the latter deformed at only $10^{\circ} \mathrm{C}$ above its $\mathrm{T}_{\mathrm{g}}$.

\section{Tilt angle}

The tilt angle $\varphi$ is defined as the acute angle that the limb of the chevron fold makes with the deformation direction. It represents the degree of tilting of lamellae and shows how sharp the chevron pattern is. In earlier works the interlimb angle $\psi$ is used, corresponding to twice the tilt angle, i.e. $\psi=2 \varphi$. The tilt angle changes with the strain and the temperature at which the deformation is performed. We followed this changes performing deformations of the semicrystalline polymers at different temperatures.

According to the theories of undulation instability above a critical stress the multilayered materials buckle, with an initially sinusoidal buckling profile which further transforms to chevron pattern. TEM do not allow to observe the buckling process "in situ". The micrographs show the final result after the deformation is completed. In order to check if this mechanism applies also for semicrystalline polymers we tried to perform investigation at conditions near to the critical ones, where the undulation starts.

For a semicrystalline polymer a critical region is around the glass transition $\mathrm{T}_{\mathrm{g}}$, since below $\mathrm{T}_{\mathrm{g}}$ folding is hindered, according to the discussion in $\S 3.1$. Thus we studied the deformation structures in sPS at temperature of $100^{\circ} \mathrm{C}$, just around the glass transition of $98^{\circ} \mathrm{C}$ (measured by DSC). As can be seen in Figure 11, folds have formed, but they have rather smooth curved shape, than sharp hinges. In first approximation they can be regarded as undulations of sinusoidal form, supporting herewith the theory of undulation instability. Further, if the transformation of the initial sinusoidal folds goes trough concentric-like to chevron folds, 
vestiges of the earlier concentric-like pattern should also be observable. We believe to detect them also in this sPS sample, on the right side of Figure 11.

On the other hand, the observed undulations are not very regular and less coherent in wavelength and amplitude, and in these features remind the pictures found in SBS block copolymers at strains below $20 \%$ [15]. In the cited work these observations are discussed as indications of the alternative mechanism of folding, assuming first kink nucleation at the defects and evolution to chevron morphology through further propagation of the kink boundaries into adjacent regions.

Obviously, our current observations are not sufficient for a definitive statement about the relative importance of these two mechanisms in semicrystalline polymers. It could be that both mechanisms are possible, but which will take place depends on the specific microstructure of the semicrystalline polymer.

In the regions where the chevron shape is well formed, the tilt angle can be evaluated. Near the $T_{g}$ it is relatively large, about $70^{\circ}$. With increasing deformation temperature the chevrons rapidly become sharper, and at only $10^{\circ} \mathrm{C}$ above the glass transition of sPS the average tilt angle amounts already $55^{\circ}$. The folds have become regular and coherent in wavelength, as depicted in Figures 6.

A comparison of the chevron patterns in different polymers shows a great variety of tilt angles: for $\beta$-iPP upon deformation at room temperature the typical tilt angle is $36-40^{\circ}$, in HDPE the limbs close to an average angle of $30^{\circ}$. In general, a trend of decreasing tilt angles with increasing difference $\Delta T$ between deformation temperature and glass transition is observed. The difference $\Delta T=T_{\text {def. }}-T_{g}$ accounts indirectly for compliance, mobility and viscosity of the soft phase, all of them being factors which affect the formation of chevron morphology under force. However the plot of the tilt angle in the different polymers as a function of $\Delta T$ is not a straight line. This result can be related to the complex influence of the microstructural details of each polymer on the chevron formation.

Another point is the dependence of tilt angle on deformation strain. For other layered systems it is shown that with increasing strain the chevrons become sharper, i.e. the tilt angle $\varphi$ between lamellae and deformation direction decreases. For a globally oriented lamellar SBS block copolymer a model of affine tilting of lamellae with macroscopic elongation $\lambda$ upon perpendicular deformation at room temperature was proposed [12]. Many years earlier a linear dependence of the tilt angle on the extent of roll in POM has been reported [17]. The situation in unoriented materials is expected to be more complicated. It should be mentioned however, that 
we observed nearly linear decrease of the tilt angle in the folded domains of unoriented sPS with increasing deformation. Detailed investigations in other polymers are still under way.

\section{$\S 4$. CONCLUSIONS}

(1) In this work we have demonstrated that chevron morphology can be formed under dilative strain not only in special polymeric materials with layered structure, but also in traditional semicrystalline polymers. The prerequisites therefore are layered lamellar arrangement and large difference in stiffness between the crystalline and amorphous phases.

(2) We showed that chevron folding is a common deformation mode which can appear in real, i.e. globally unoriented or partially oriented polymers, in areas where the lamellar stacks are oriented perpendicular to the deformation direction. Consequently, chevron folding in semicrystalline polymers has to be regarded as a possible microdeformation of equal importance as the three main modes generally accepted.

(3) The mechanism of chevron folding in semicrystalline polymers and the main characteristics of the chevron pattern are similar to that in other layered systems. The wavelength of the folding is affected by the surrounding lamellar domains. Accompanying details as simultaneous void formation, asymmetry etc. are influenced by the specific microstructure of each polymer.

\section{ACKNOWLEDGEMENTS}

The authors are indebted to Ing. S.Goerlitz and Ing. I.Naumann for a significant part of the TEM investigations presented in this work. 


\section{REFERENCES}

1. JOHNSON, A.M., 1977 Styles of Folding, (Elsevier, New York)

2. RAMSAY, J.G. and HUBER, M.I., 1987, The Techniques of Modern Structural Geology (Academic, London), Vol. 2;

3. MOLHO, P., PORTESEIL J.L., SOUCHE Y., GOUZERH J. and LEVY J.C.S., 1987, J.Appl.Phys. 61, 4188

4. SEUL, M. and WOLFE, R., 1992, Phys. Rev. Lett. 68, 2460.

5. SINGER, S.J., 1993, Phys. Rev. E 48, 2796

6. RIBOTTA, R., MEYER, R.B. and DURAND, G., 1976, J. Phys. (Paris), 35, L161.

7. RIEKER, T.P., CLARKE, N.A., SMITH, G.S., PARMAR, D.S., SIROTA, E.B. and SAFINYA, C.R., 1987, Phys. Rev. Lett. 59, 2658.

8. SHALAGINOV, A.N., HAZELWOOD, L.D. and SLUCKIN, T.J., 1998, Phys. Rev. E 58, 7455

9. FUJIMURA, M.; HASHIMOTO, T. and KAWAI, H., 1978, Rubber Chem.Technol. 51, 215.

10. SÉGUÉLA, R. and PRUD’HOMME, J., 1981, Macromolecules, 14, 197.

11. HONEKER, C.C. and THOMAS, E.L., 1996, Chem. Mater. 8, 1702

12. COHEN, Y., ALBALAK, R.J., DAIR, B.J., CAPEL, M.S. and THOMAS, E.L., 2000, Macromolecules, 33, 6502.

13. YAMAOKA, I. and KIMURA, M. 1993, Polymer, 34, 4399.

14. HUY, T.A., ADHIKARI, R. and MICHLER, G.H., 2003, Polymer 44, 1247

15. COHEN, Y., BRINKMANN, M. AND THOMAS, E.L., 2001, J. Chem. Phys., 114, 984

16. READ; D.J., DUCKETT R.A., SWEENEY, J. and MCLEISH, T.C.B., 1999, J.Phys.D: Appl.Phys., 32, 2087.

17. GEZOVICH, D.M. and GEIL, P.H., 1971, J.Mat.Sci., 6, 509

18. GEZOVICH, D.M. and GEIL, P. H., 1971, J.Mat.Sci., 6, 531

19. SONG, H.H., ARGON, A.S. and COHEN, R.E., 1990, Macromolecules, 23, 870.

20. BARTCZAK, Z., ARGON, A.S., and COHEN, R.E., 1992, Macromolecules, 25, 5036.

21. WADE ADAMS, W., YANG, D. and THOMAS, E.L., 1986, J.Mat.Sci., 21, 2239

22. HOFMANN, D, GEISS, D, JANKE, A., MICHLER, G.H. and FIEDLER, P., 1990, J.Appl. Polym.Sci., 39, 1595

23. MICHLER, G.H., MARINOW, S. and NAUMANN, I., 1989, Plaste und Kautschuk, 36, 432. 24. MICHLER, G.H. and MORAWIETZ, K., 1991, Acta Polymerica, 42, 620.

25. MICHLER, G.H., 1992, Coll. Polym. Sci., 270, 627 
26. LI, J.X., CHEUNG, W.L. and CHAN, C.M., 1999, Polymer, 40, 3641

27. MICHLER, G.H., ADHIKARI R. and HENNING S., 2004, J.Mat.Sci., 39, 3281

28. HENNING, S., ADHIKARI, R., MICHLER, G.H., BALTÁ CALLEJA, F.J. and KARGERKOCSIS, J., 2004, Macromol. Symp., 214, 157

29. Henning, S, MiChler, G.H., ANIA, F. and BAltá CALleJA, F.J., Coll. Polym. Sci., published online 1 December 2004

30 MICHLER, G.H., 1992, Kunststoff-Mikromechanik (Hanser Verlag, München, 1992)

31. LIU T., PETERMANN J., HE, CH., LIU, Z. and CHUNG T.S., 2001, Macromolecules, 34, 4305

32. EDWARDS, B.C. and PHILLIPS, P.J., 1974, Polymer, 15, 351

33. CRETON, C., KRAMER, E.J. AND HADJIIONNAU, G., 1992, Coll.Polym.Sci., 270, 399

34. GALESKI, A., 2003, Prog. Polym. Sci. 28, 1643

35. KAUSCH, H.H., GENSLER, R., GREIN, CH., PLUMMER, C.J.G. and SCARAMUZZINO, P., 1999 J.Macromol.Sci.-Phys. B38, 803

36. BAYLY, M.B., 1974, Tectonophysics, 24, 291.

37. BOWDEN, P.B. and YOUNG, R.J., 1971, Natur, 229, 23.

38. WANG, Z.G., 1994, J. Chem. Phys. 100, 2298

39. KAWAI, H. and HASHIMOTO, T. 1980, J. Macromol. Sci., B17, 427.

40. KRUMOVA, M., SEYDEWITZ, V. and MICHLER, G.H., 2002, Int. Conf. Polymer materials P2002, Halle (Saale), Sept. 2002

41. KAUFMANN, W.E. and SCHULTZ, J.M., 1973, J.Mat.Sci., 8, 41

42. PETERMANN, J. and SCHULTZ, J.M., 1978, J. Mat. Sci., 13, 50

43. HISS, R., HOBEIKA, S., LYNN, C. and STROBL, G. 1999, Macromolecules, 32, 4390

44. FIEDLER, P., MICHLER, G.H. and BRAUN, D., 1986, Acta Polym. 37, 241

45. TIMOSHENKO; S, 1988, Theory of elastic stability (McGraw Hill, New York)

46. HU, Y., HILTNER A. and BAER, E., 2004, Polym. Comp., 25, 653

47. RAMBERG, H., 1963, Bull .Am .Assoc. Pet. Geol., 47, 484

48. PATERSON, M.S. and WEISS, L.E., 1966, Geol. Soc. Am. Bull., 77, 343 


\section{FIGURE CAPTIONS}

Figure 1. Morphology of iPP: a) layered lamella arrangement in $\beta$-iPP; b) "cross-hatched" arrangement of the crystalline lamellae in $\alpha$-iPP (selectively stained ultrathin sections, TEM)

Figure 2. Chevron morphology in deformed $\beta$-iPP, deformation direction horizontal.

Figure 3. Disordered lamellar arrangement in LDPE (selectively stained ultrathin sections, TEM)

Figure 4. Lamella folding in HDPE-blow foils upon additional drawing, deformation direction vertical

Figure 5. Morphology of syndiotactic polystyrene (sPS), (selectively stained ultrathin sections, TEM)

Figure 6. Chevron structure in SPS after tensile deformation at $110^{\circ} \mathrm{C}$, deformation direction shown by the arrow

Figure 7. Schematic representation of the morphology of globally unoriented semicrystalline polymer: before deformation (top) and after deformation (bottom)

Figure 8. Coexistence of chevron pattern and microvoids in deformed samples of $\beta$-iPP, deformation direction horizontal

Figure 9. Chevron folds with different wavelengths in a sample of sPS, deformation direction shown by the arrow

Figure 10. Chevron pattern in deformed SBS block copolymer with lamellar morphology, deformation direction shown by the arrow

Figure 11. Morphology of sPS formed upon deformation at temperature around the glass transition, deformation direction shown by the arrow 


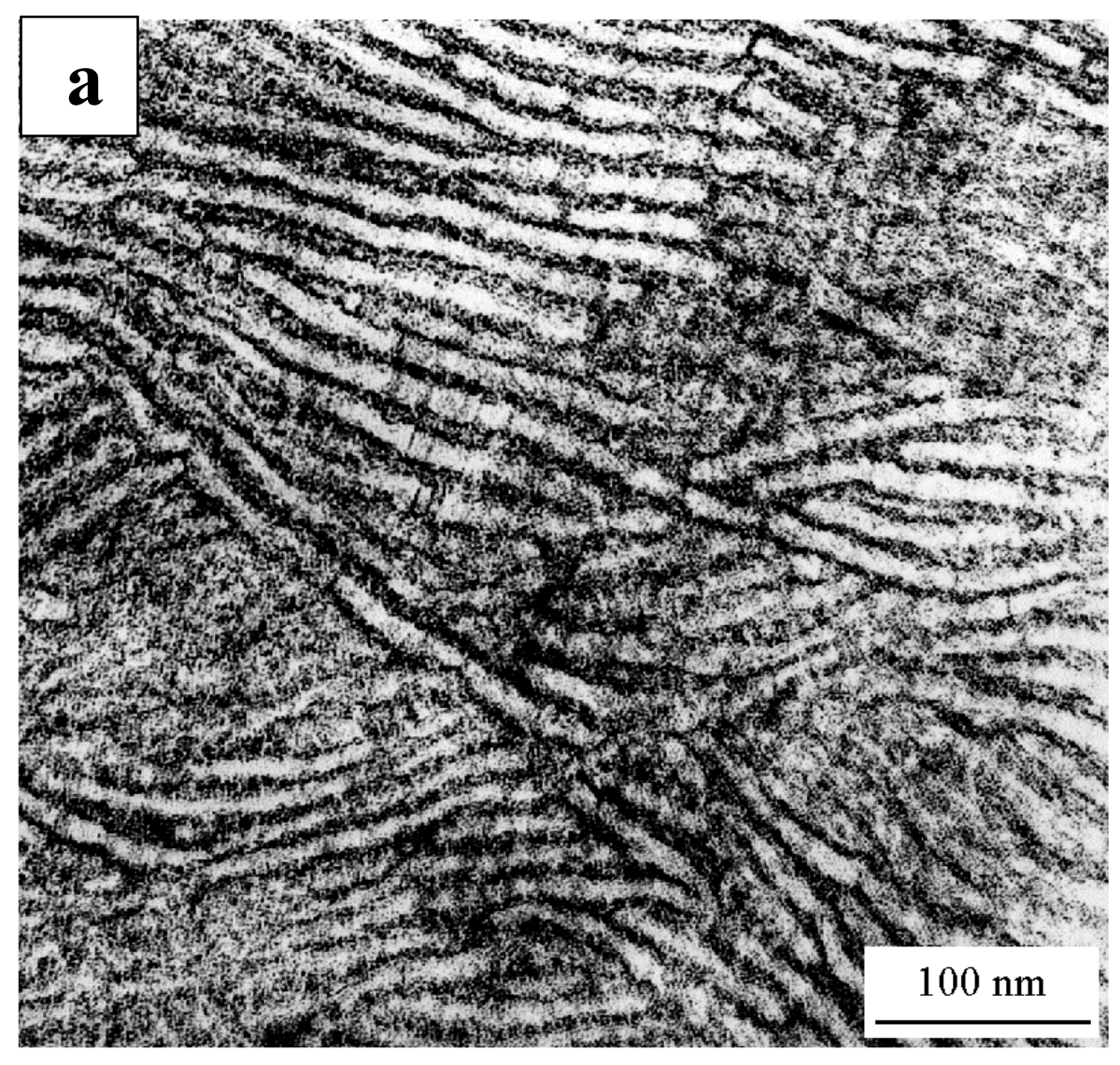

$\beta$

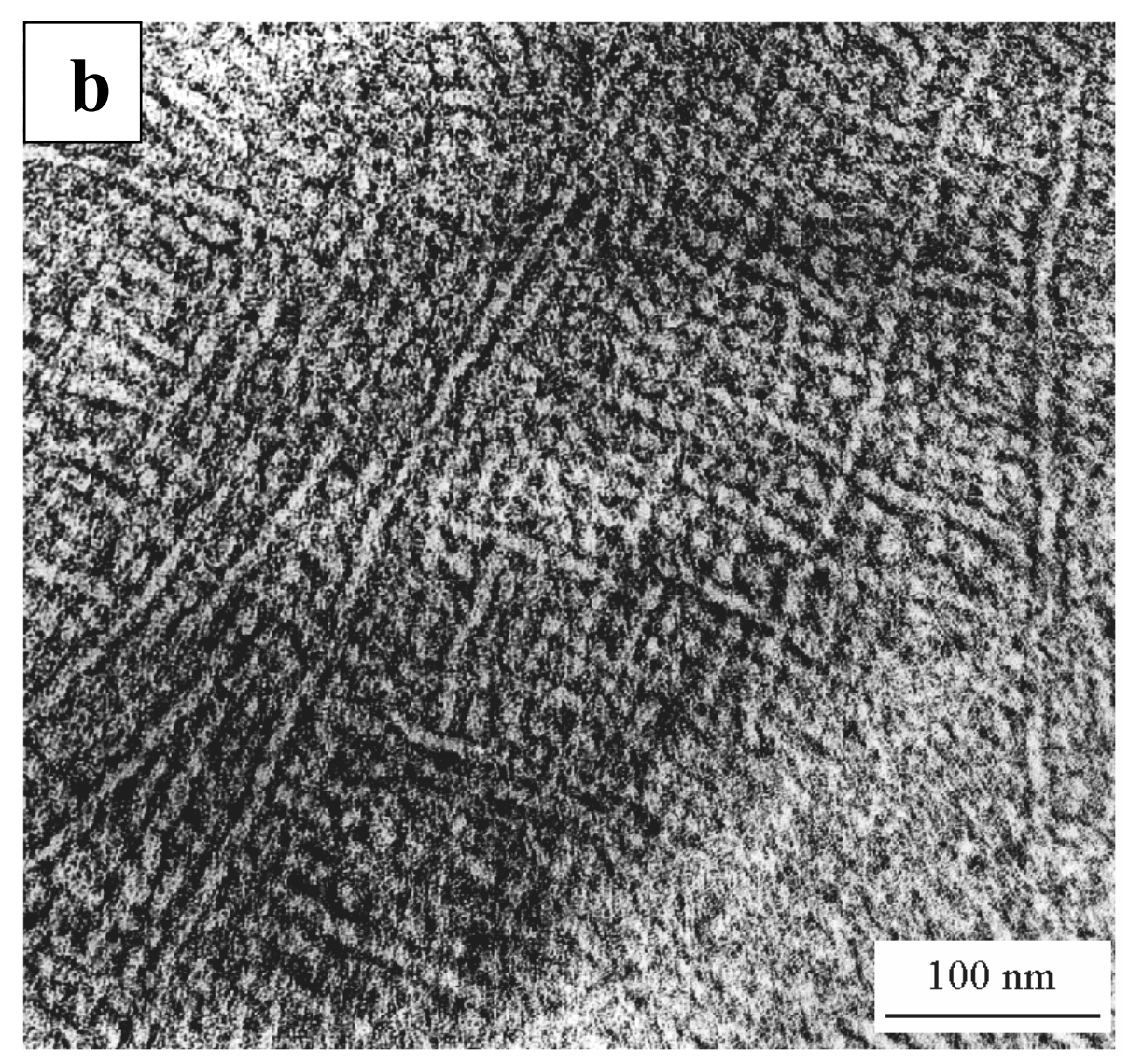

$\alpha$

Fig. 1 


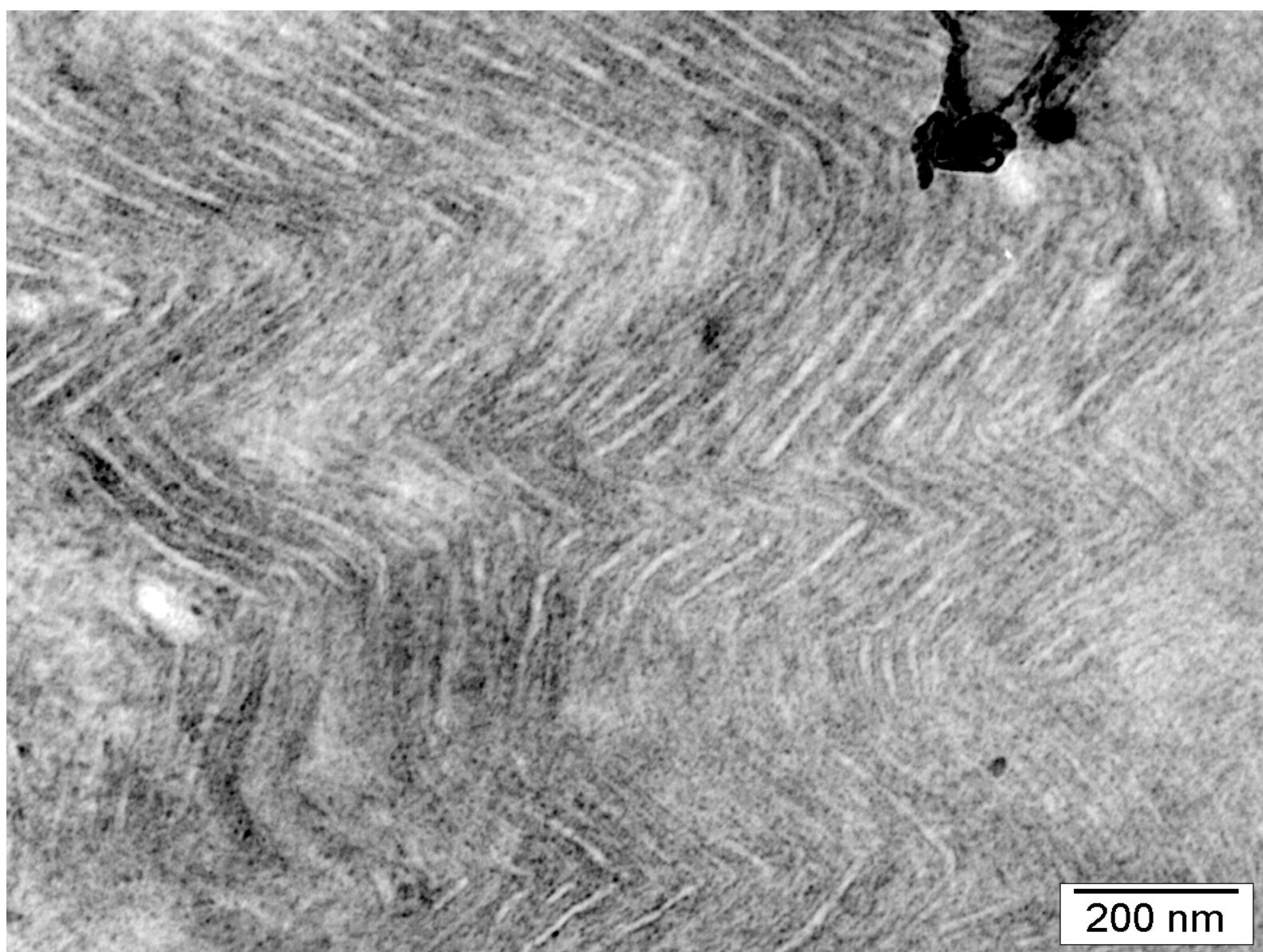

Fig. 2 


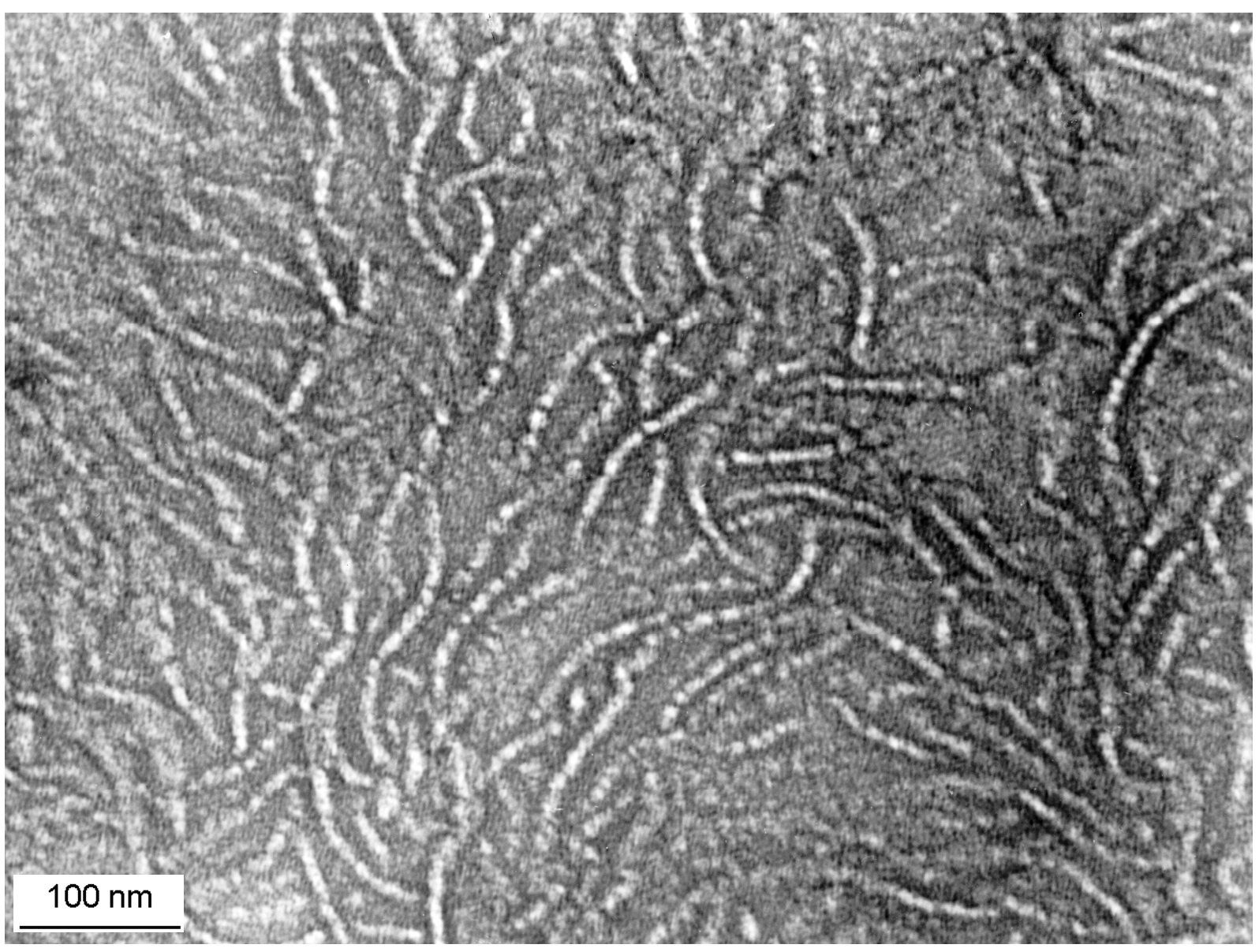

Fig. 3 


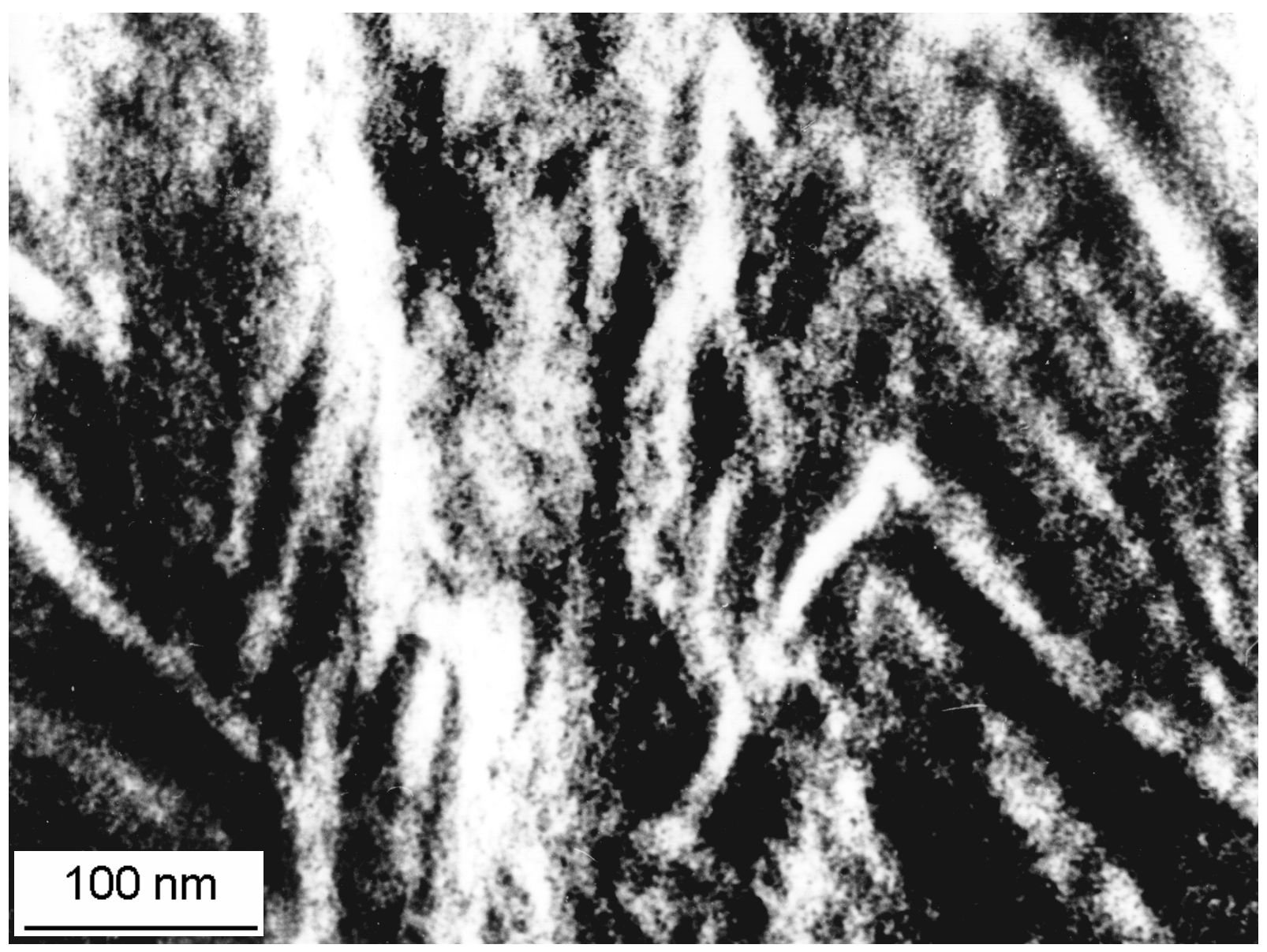

Fig. 4 


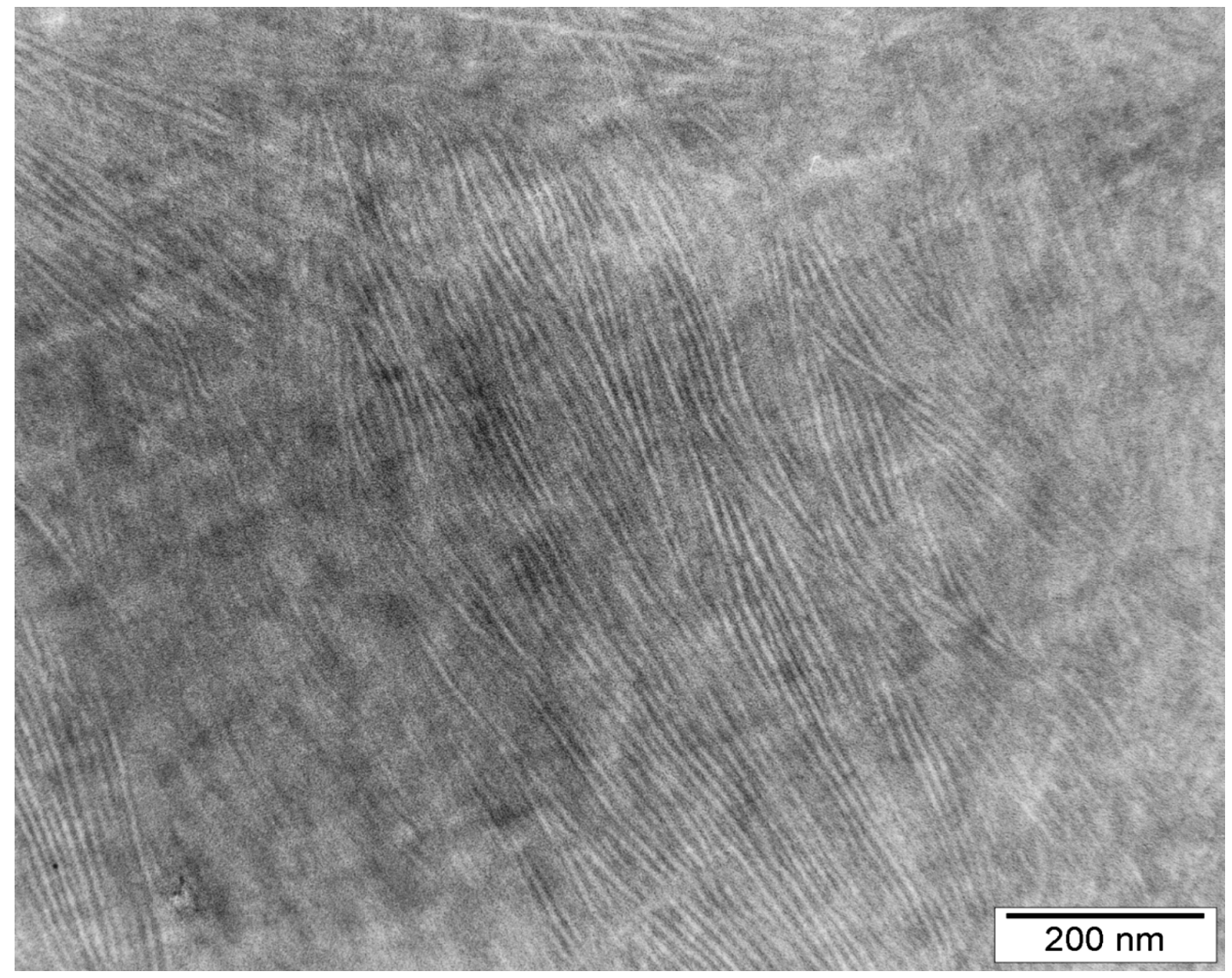

Fig. 5 


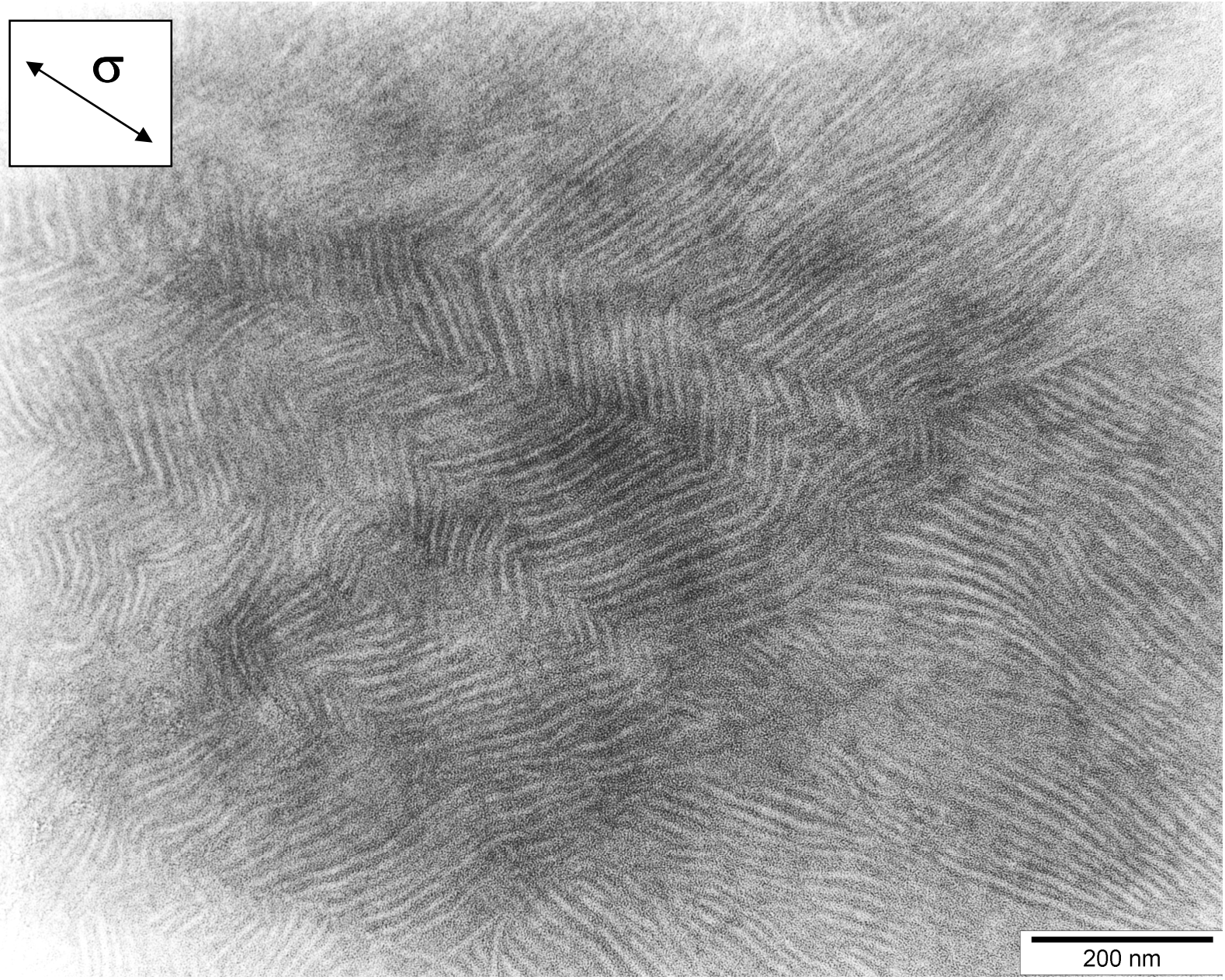

Fig. 6 

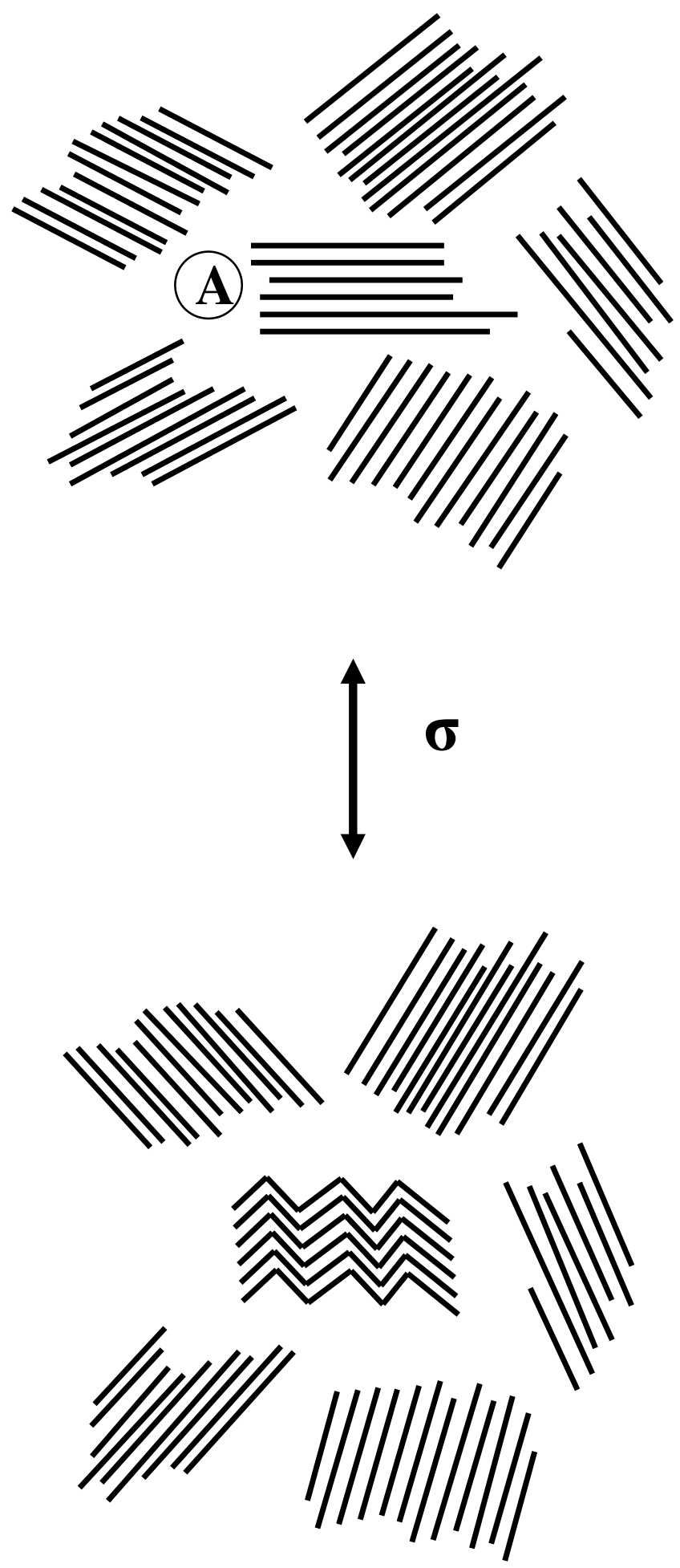

Fig. 7 

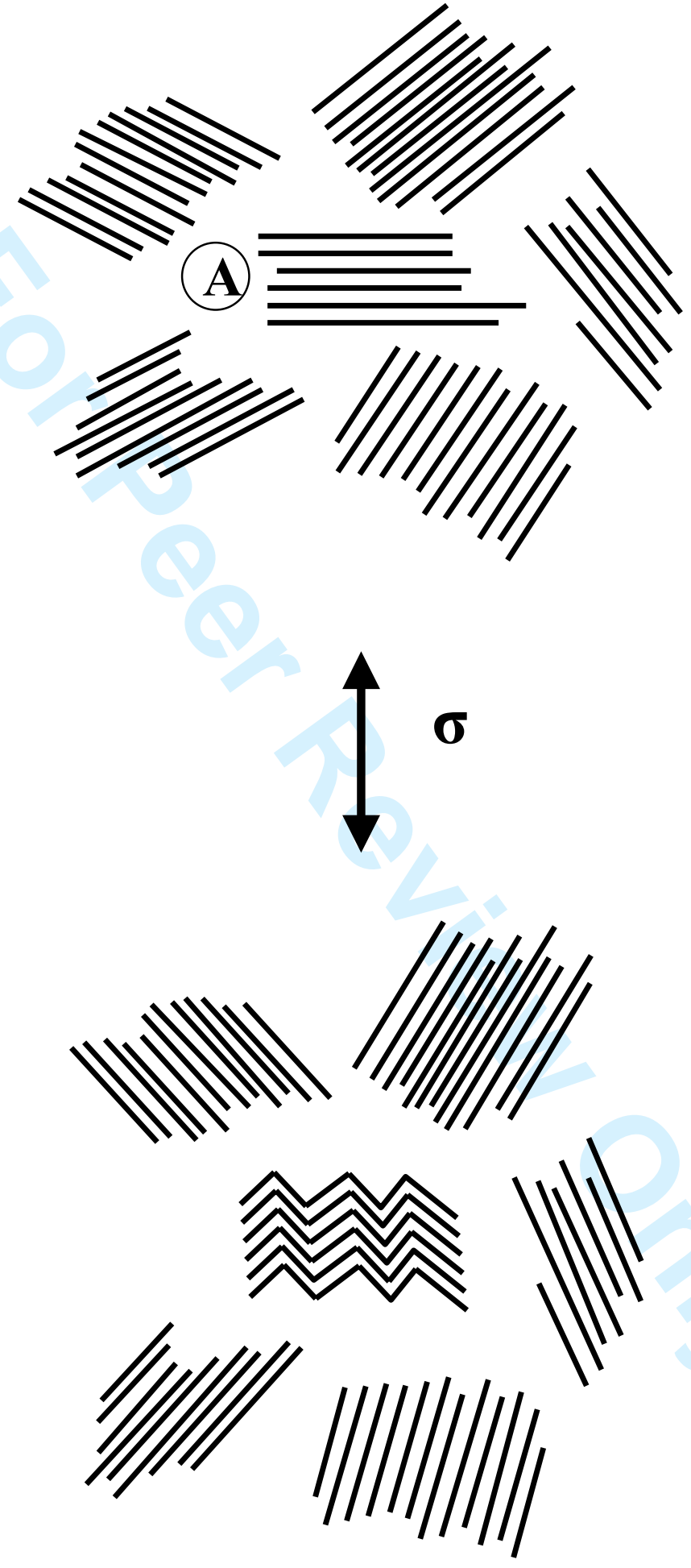

Fig. 7 


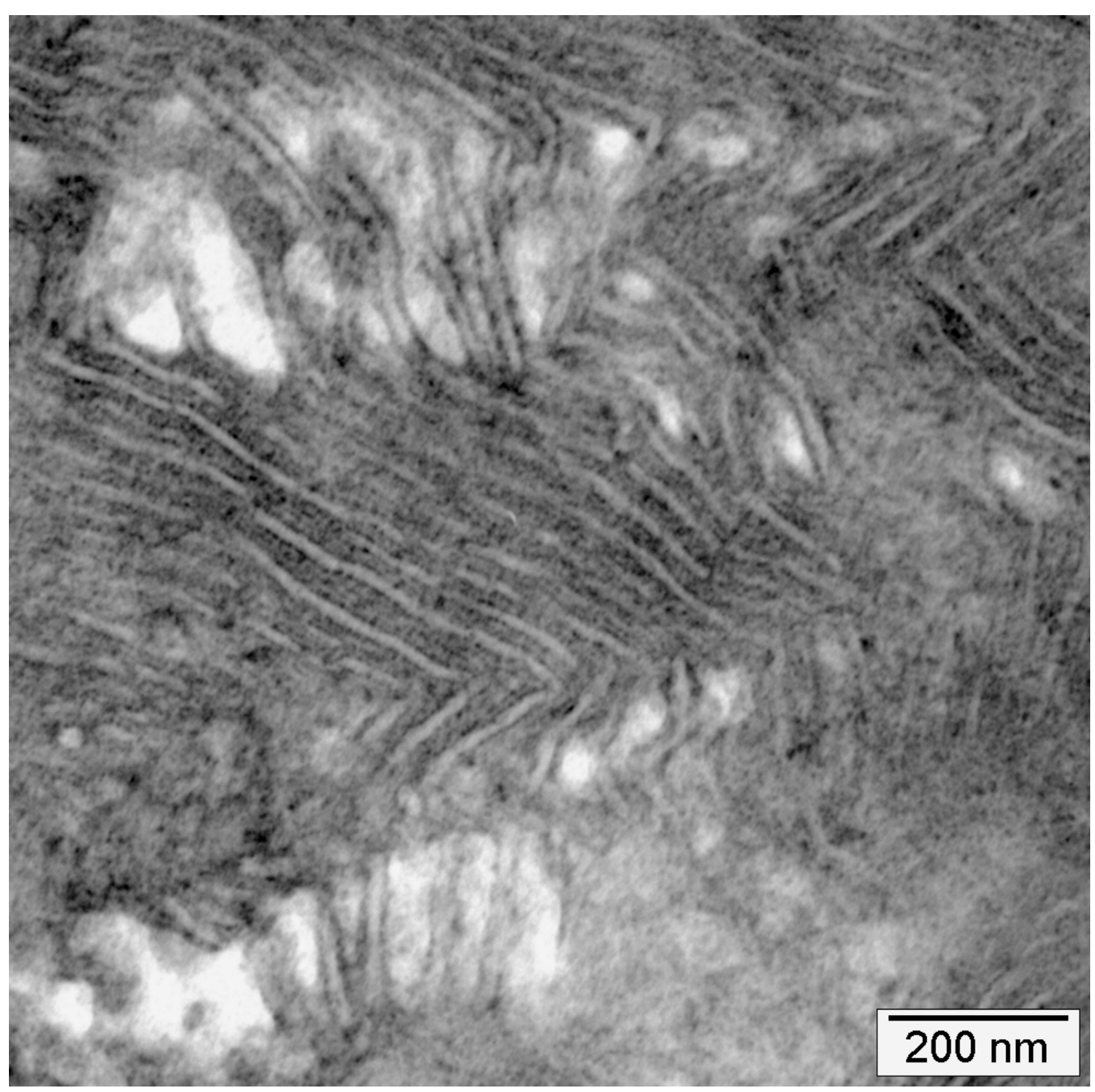

Fig. 8 


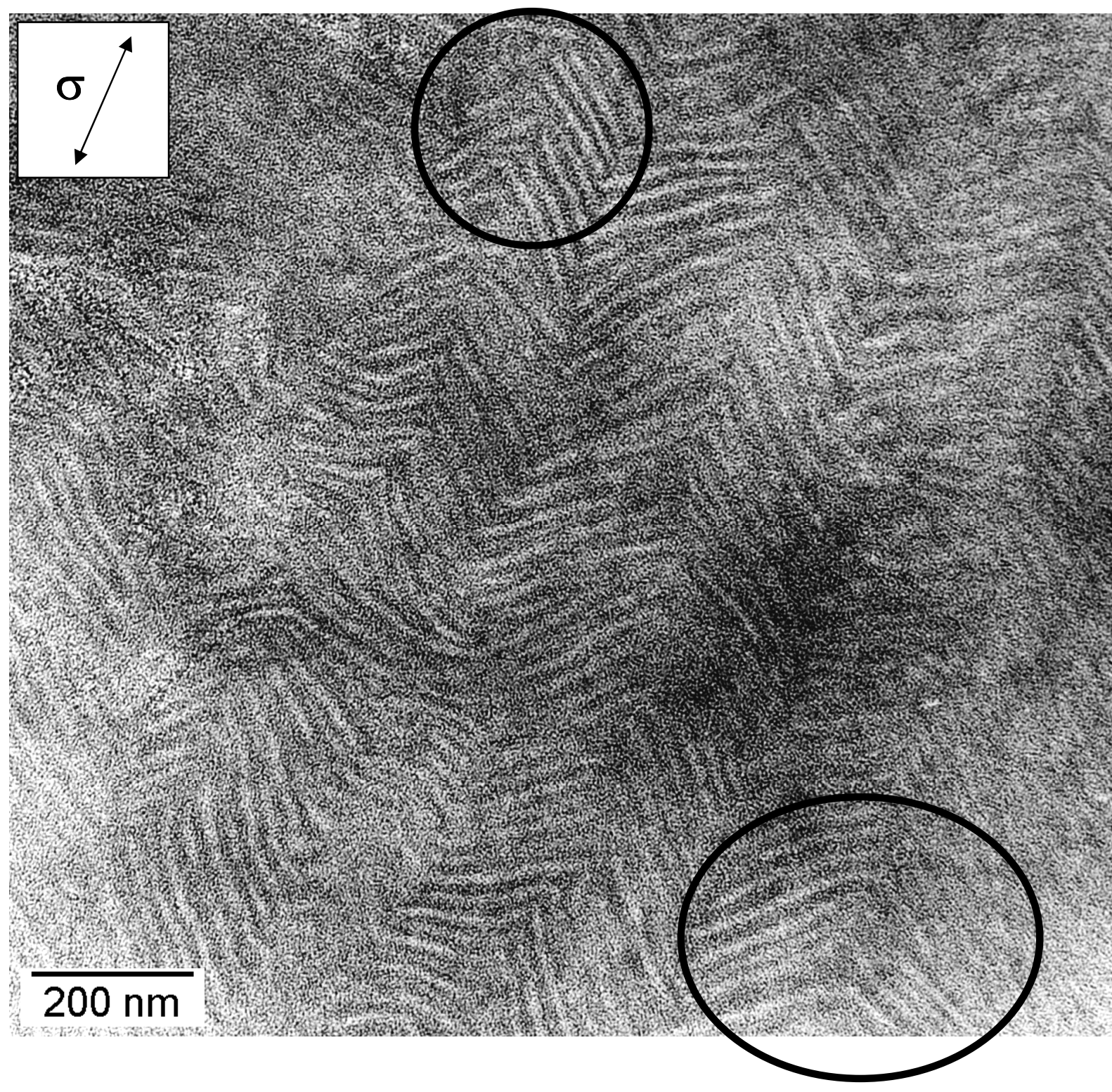

Fig. 9

http://mc.manuscriptcentral.com/pm-pml 


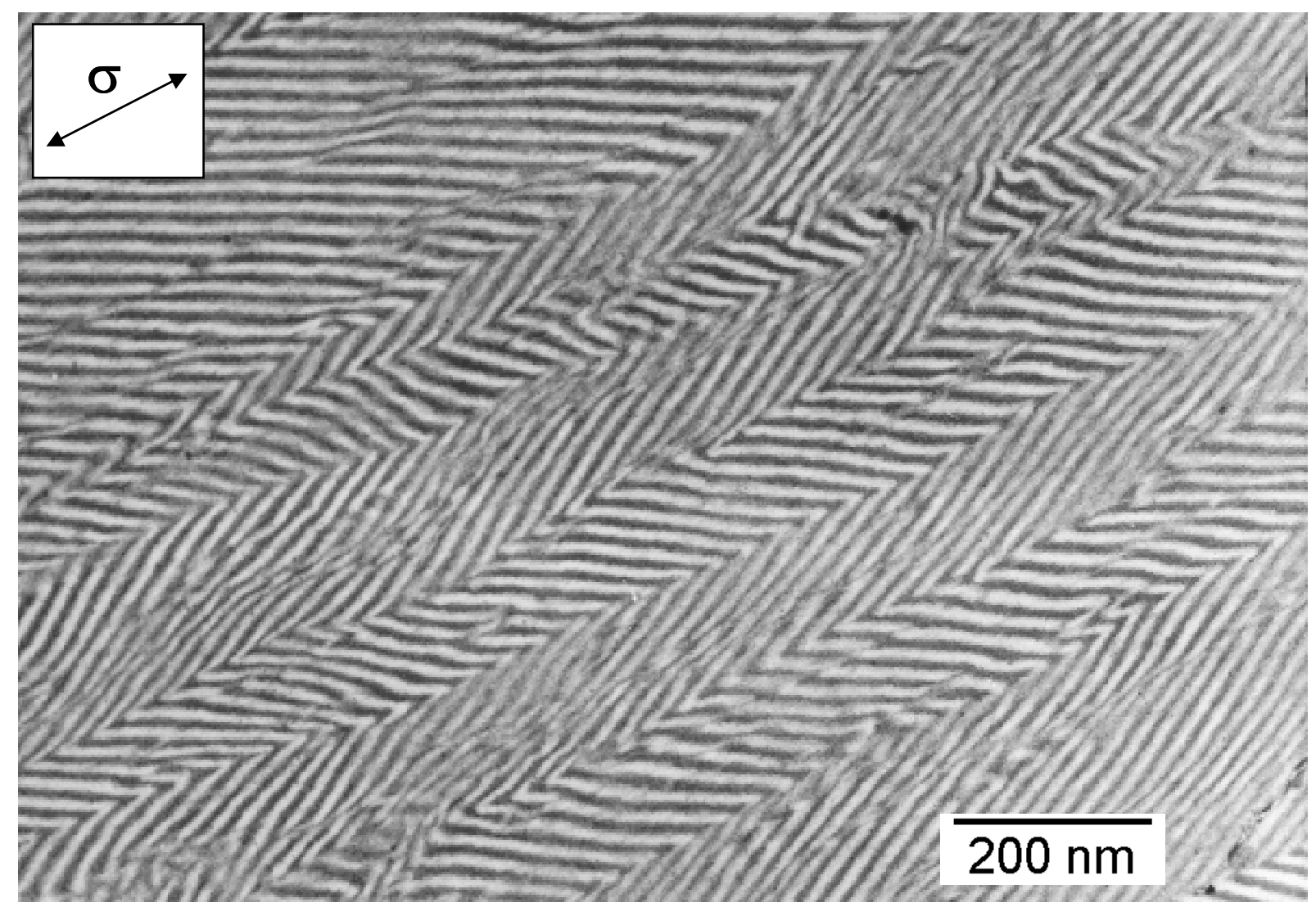

Fig. 10 


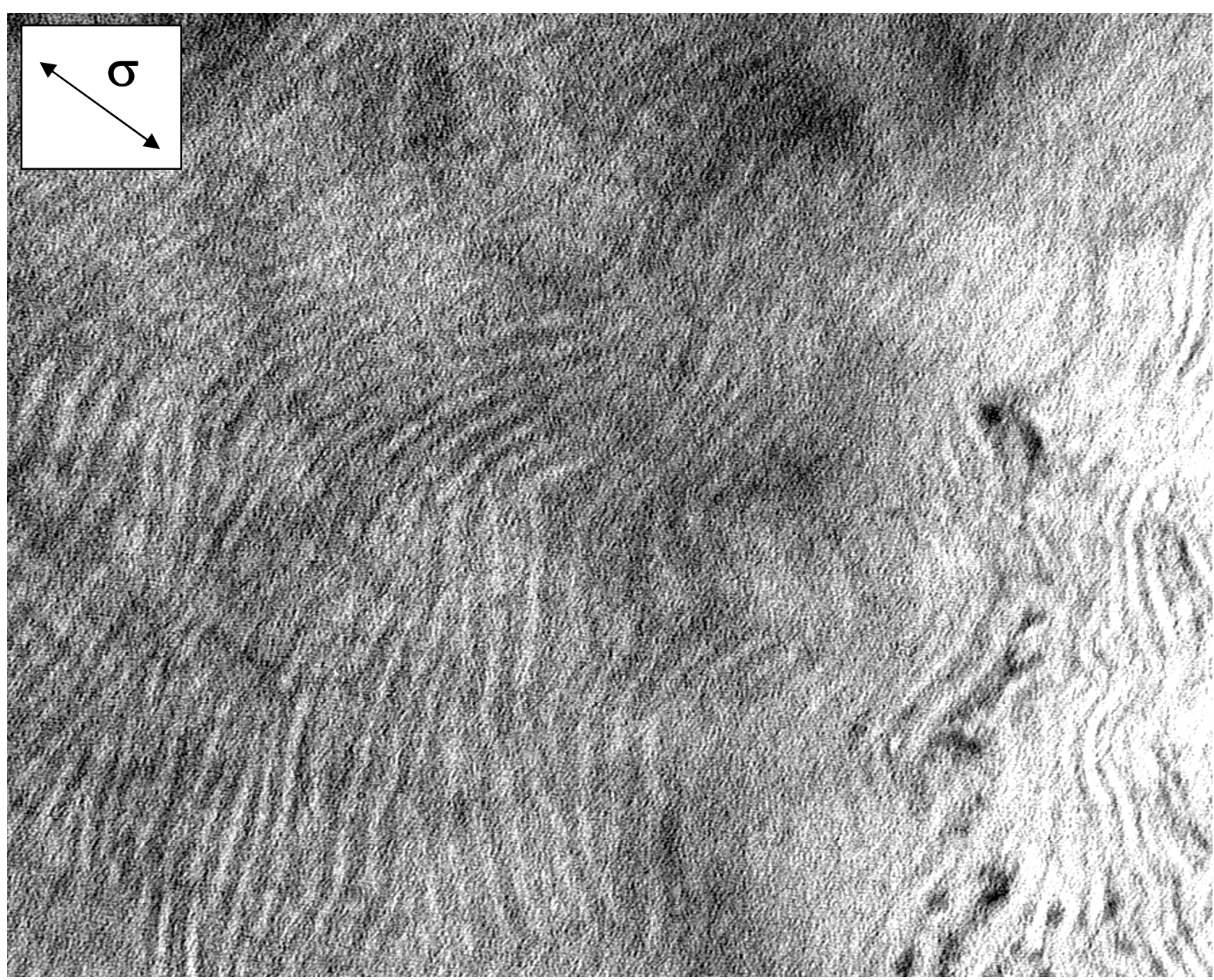

Fig. 11 


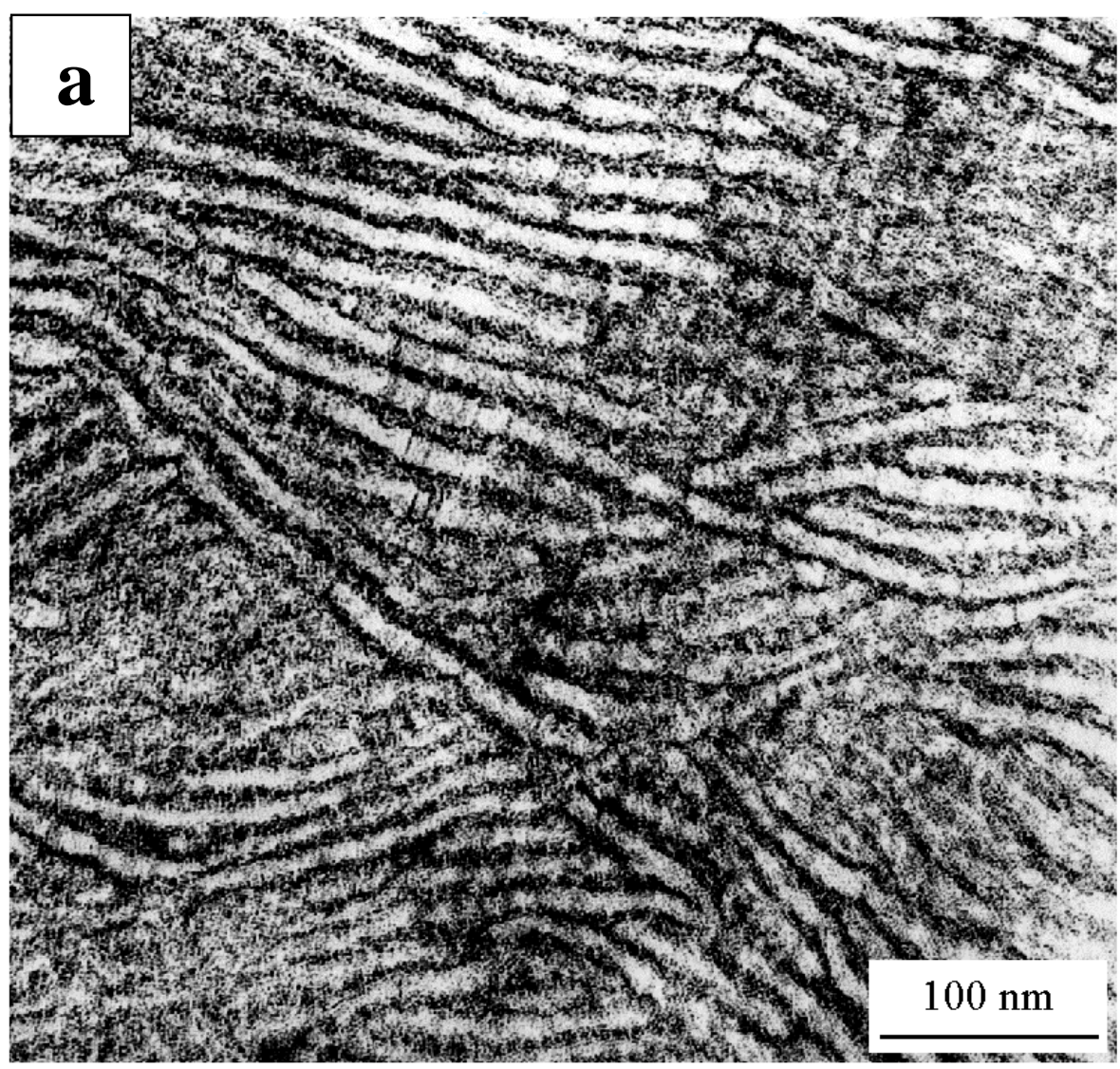

$\beta$

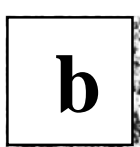

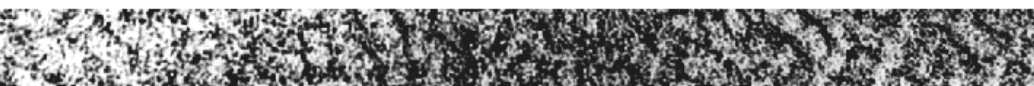

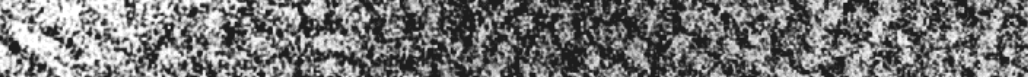

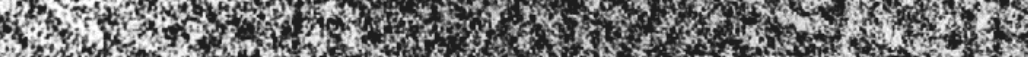

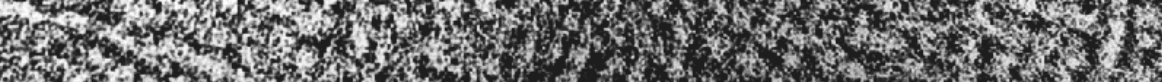
r.7.7. 6.5.5. F,

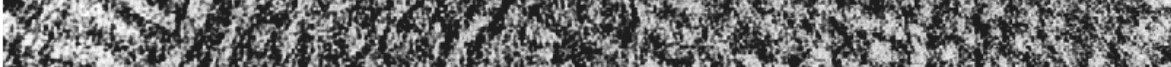
167,

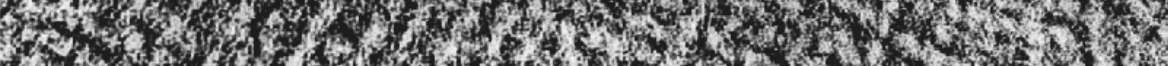

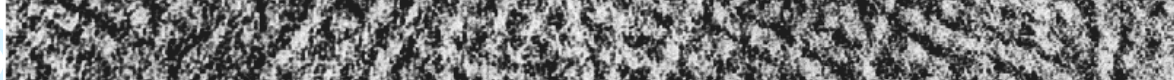

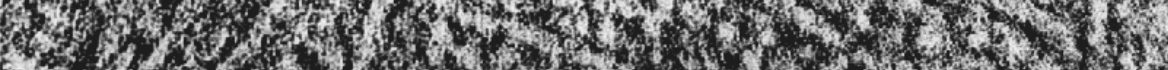

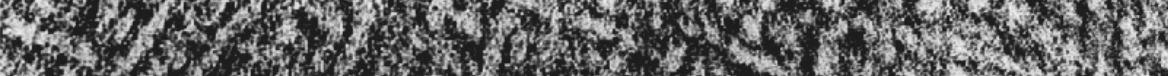

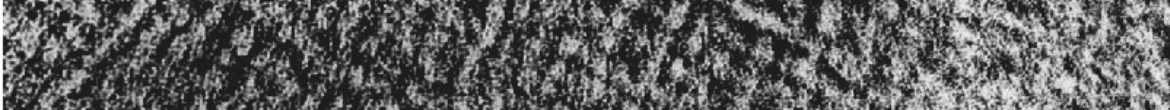

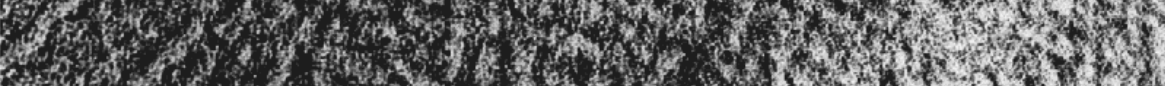

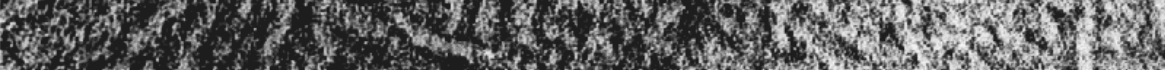

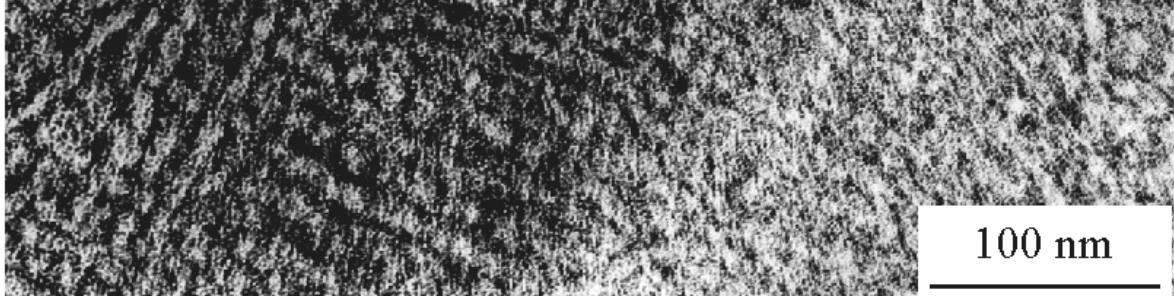
3. $100 \mathrm{~nm}$

$\alpha$

Fig. 1 


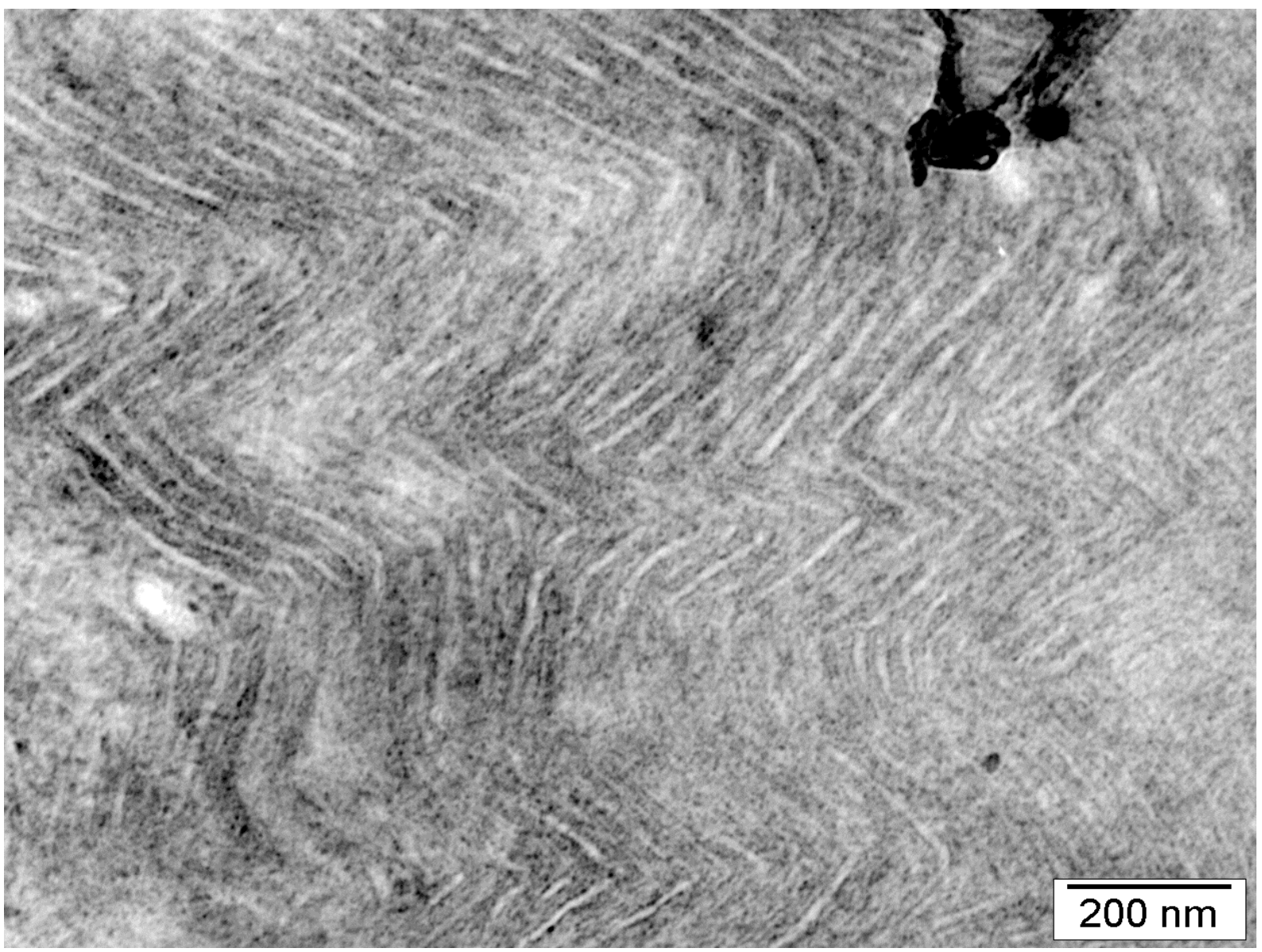

Fig. 2 


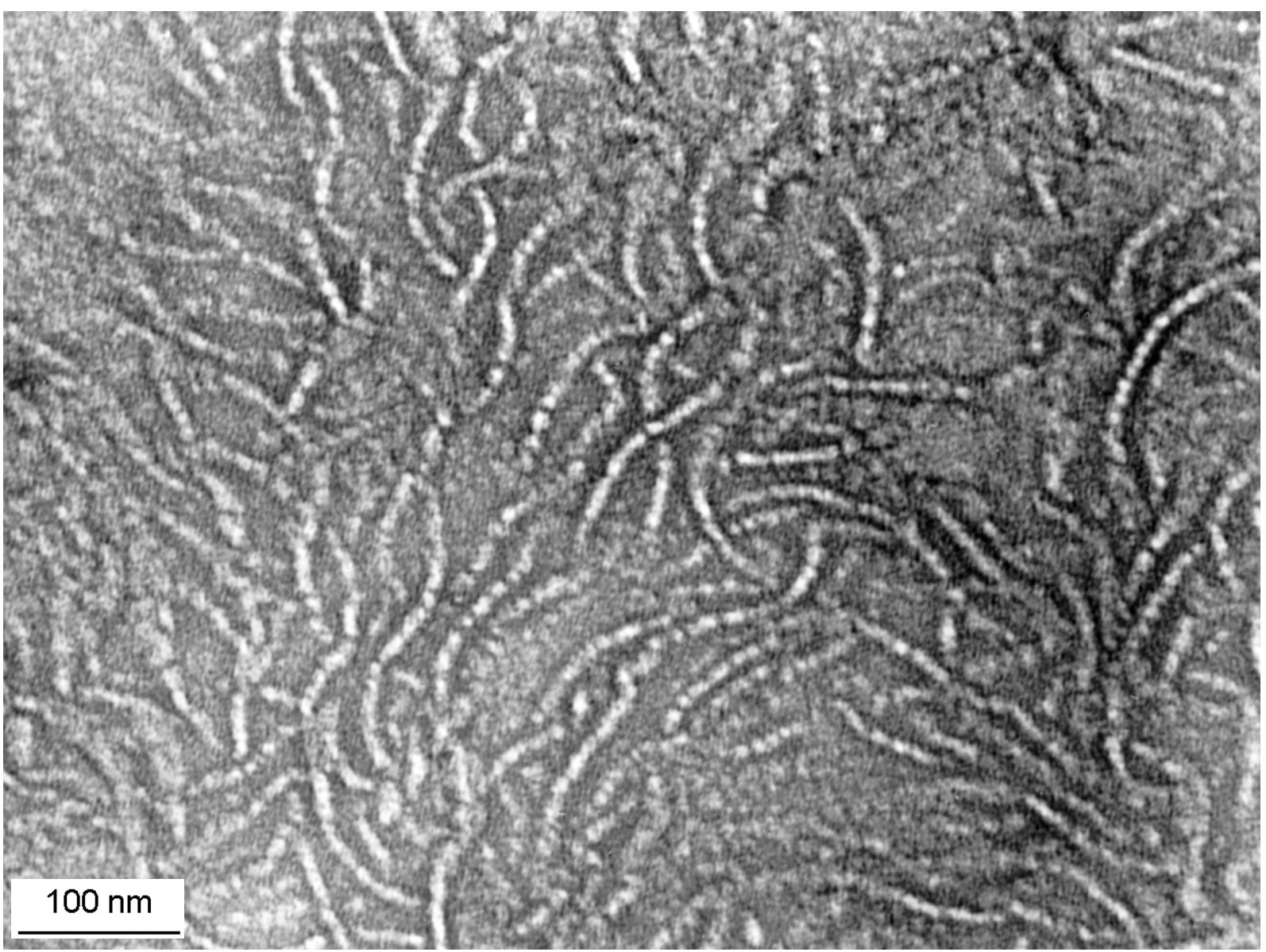

Fig. 3 


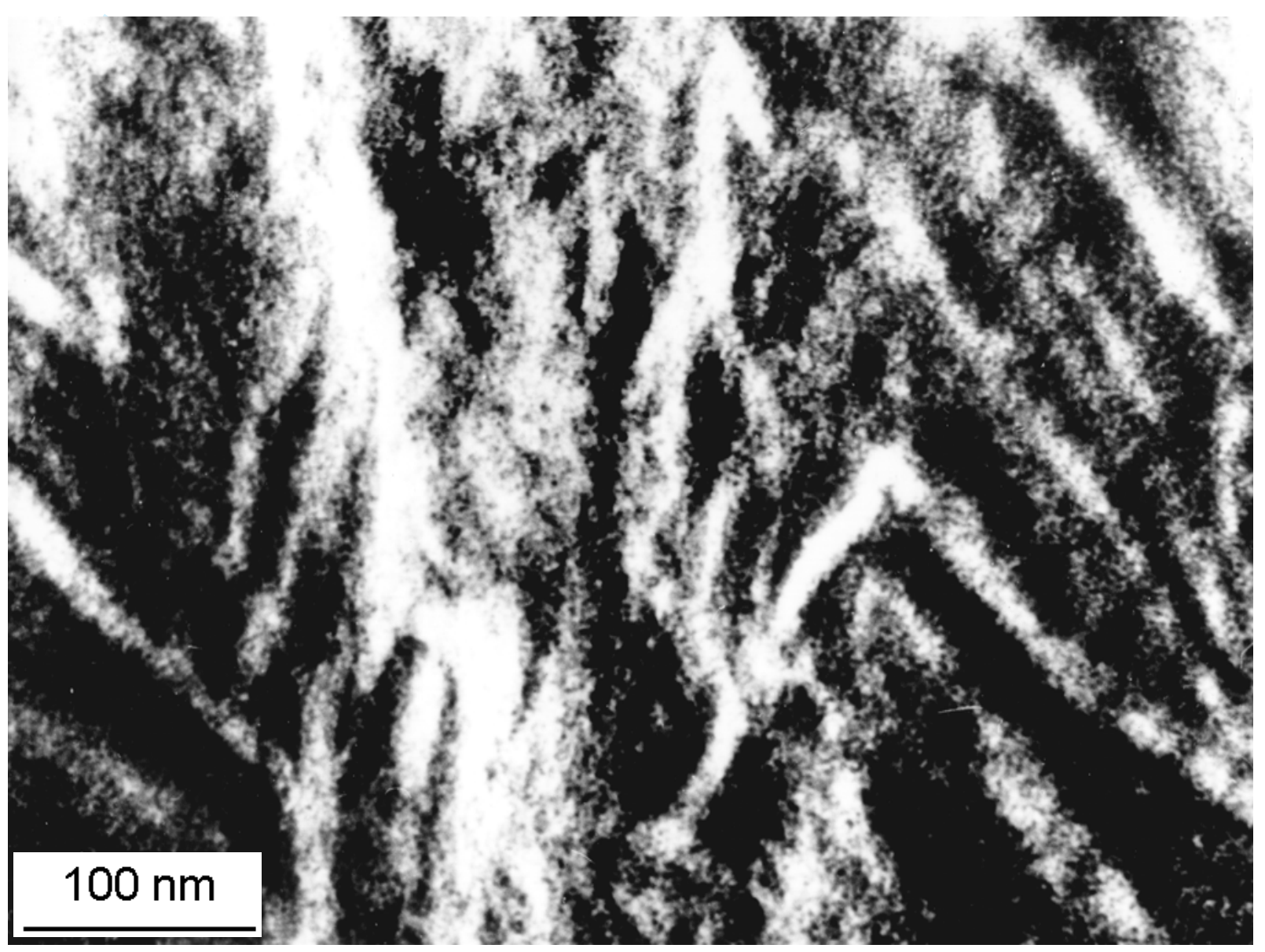

Fig. 4 


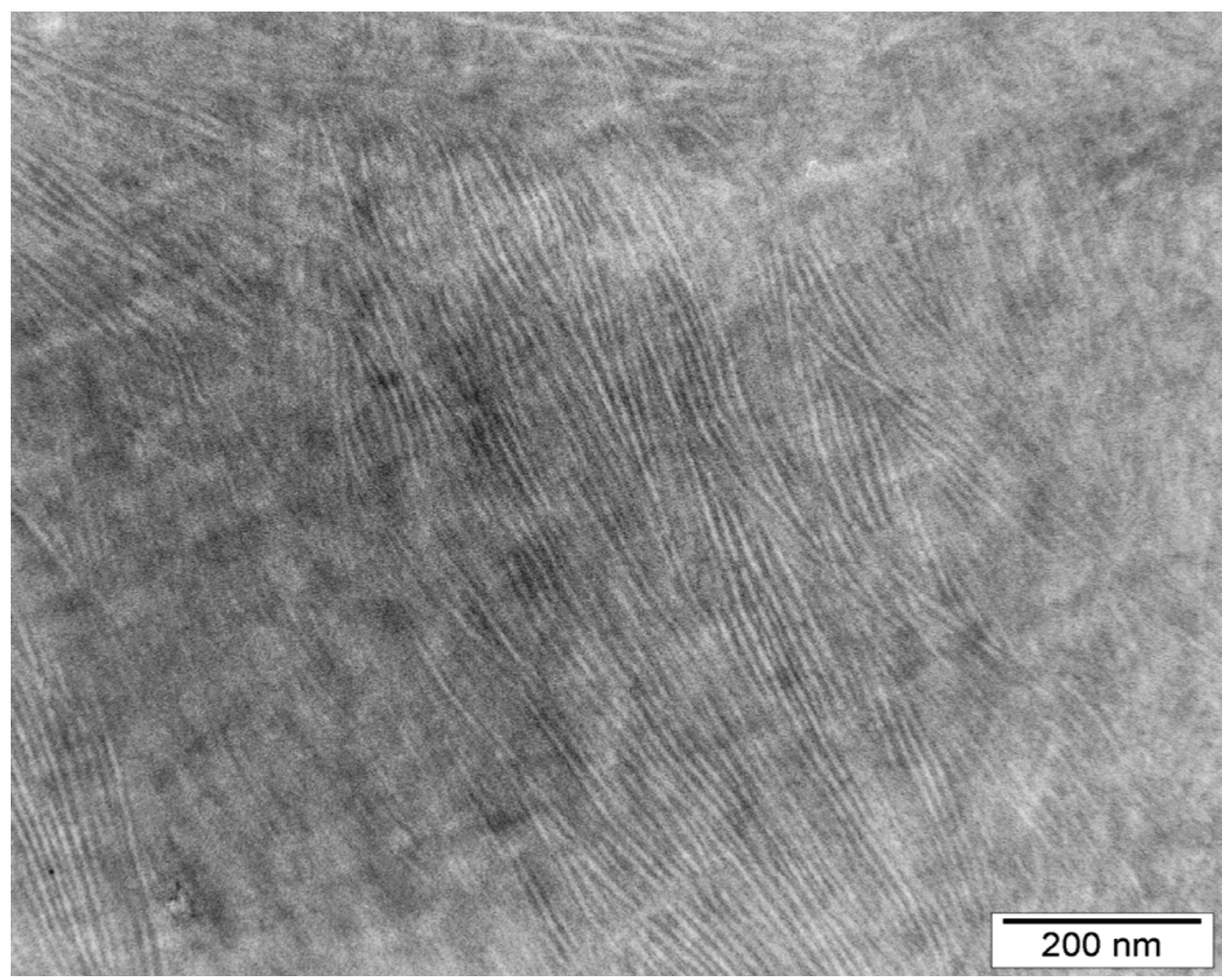

Fig. 5 


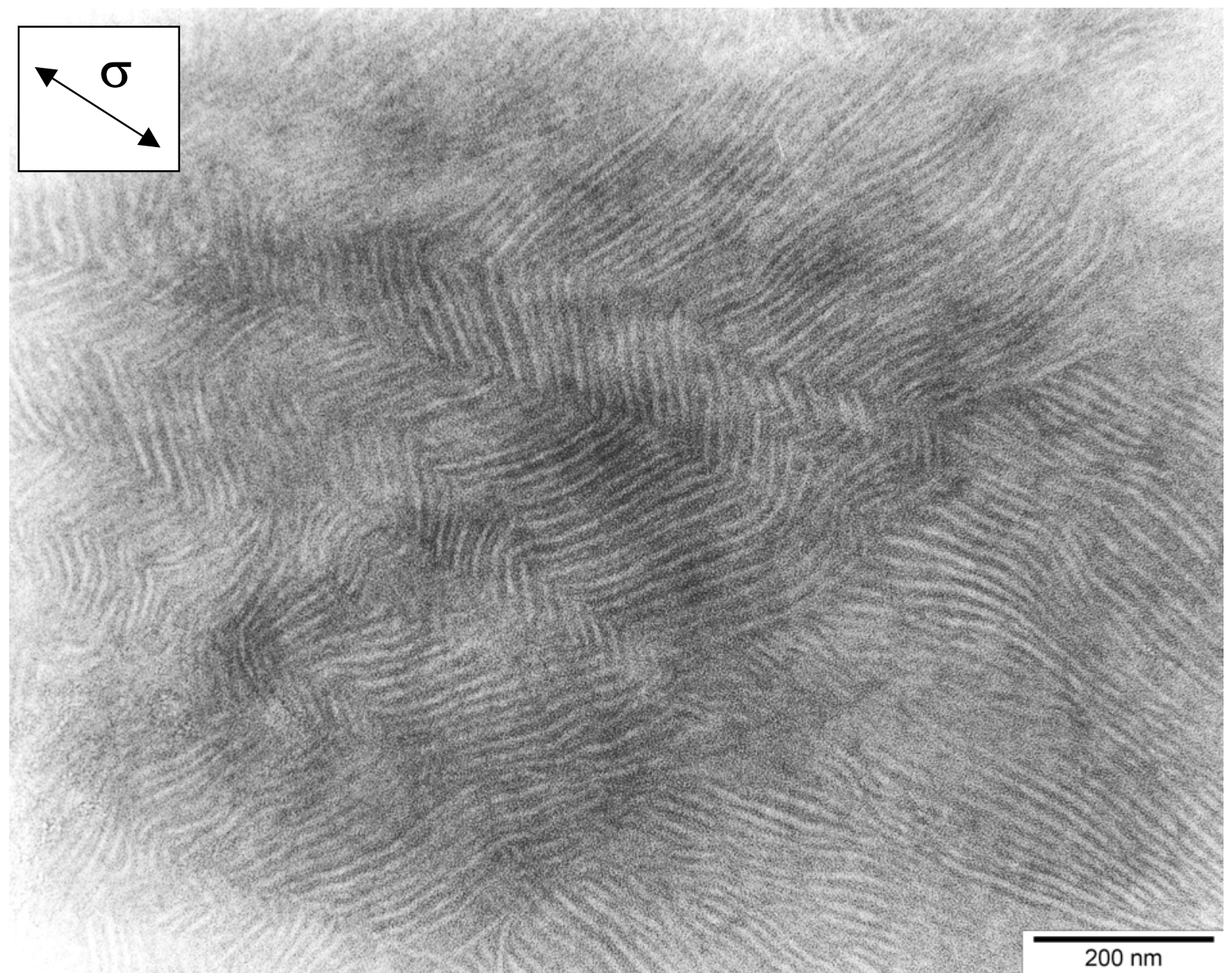

Fig. 6 


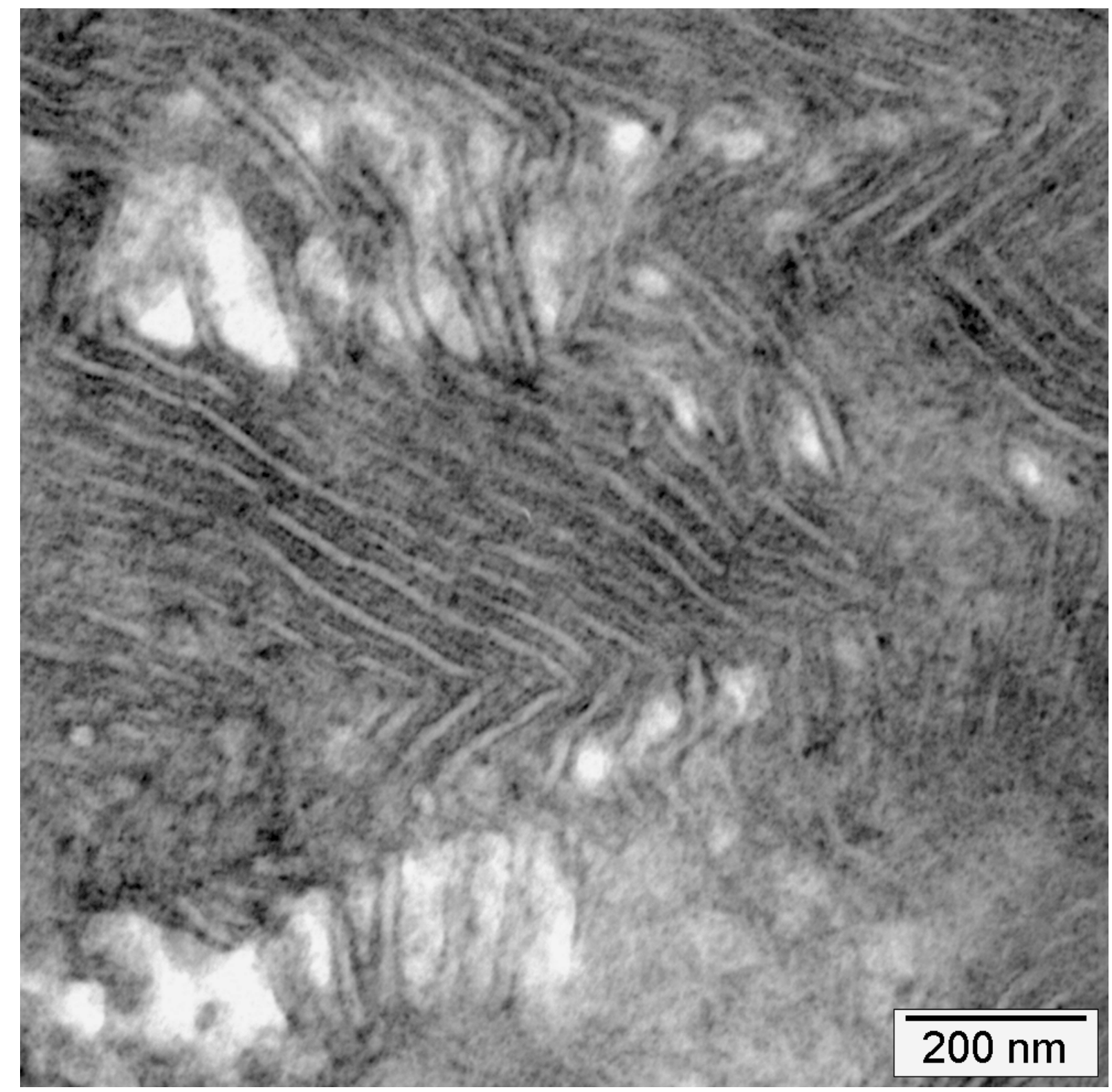

Fig. 8 
Fig. 9

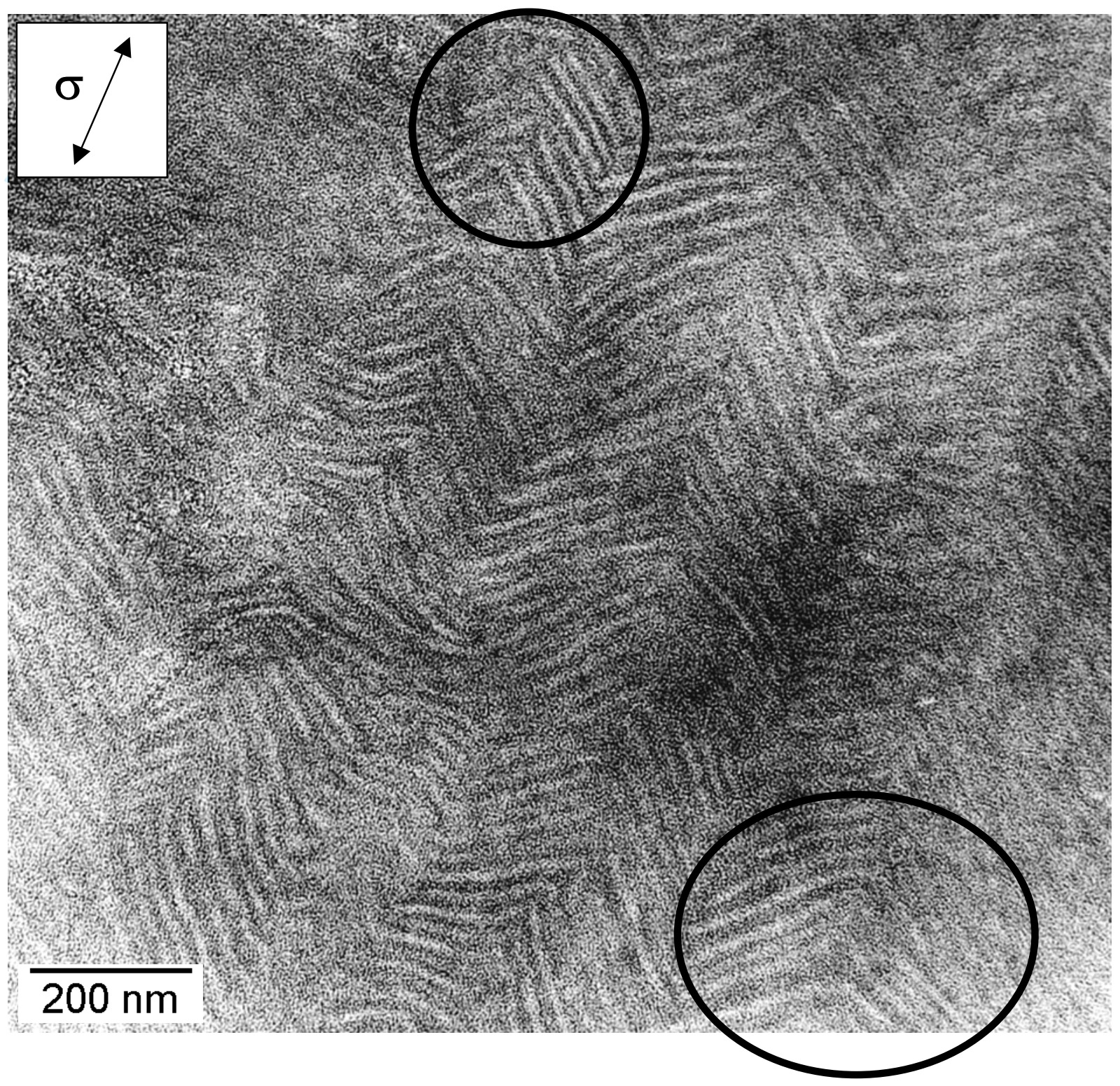

http://mc.manuscriptcentral.com/pm-pml 


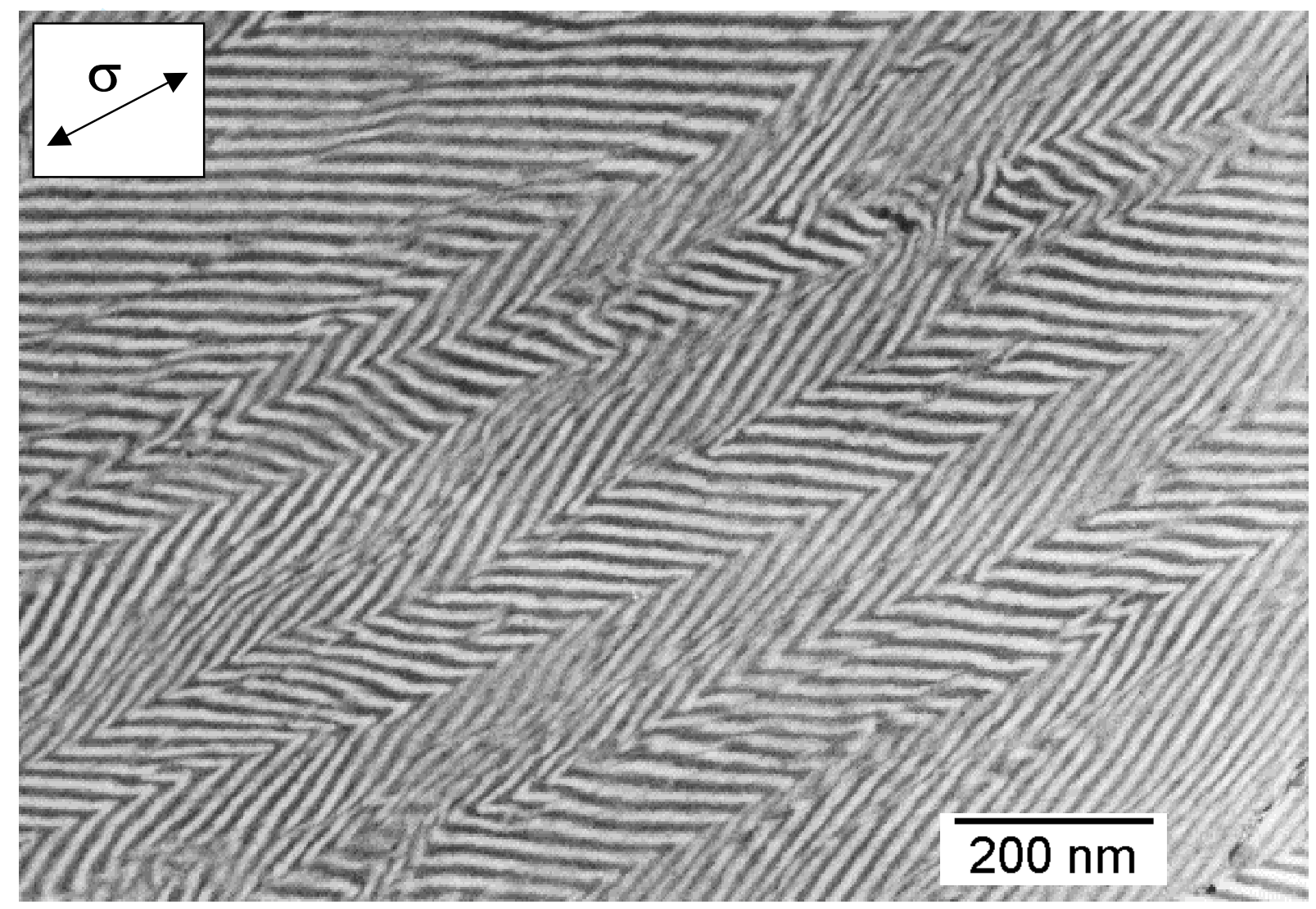

Fig. 10

http://mc.manuscriptcentral.com/pm-pml 


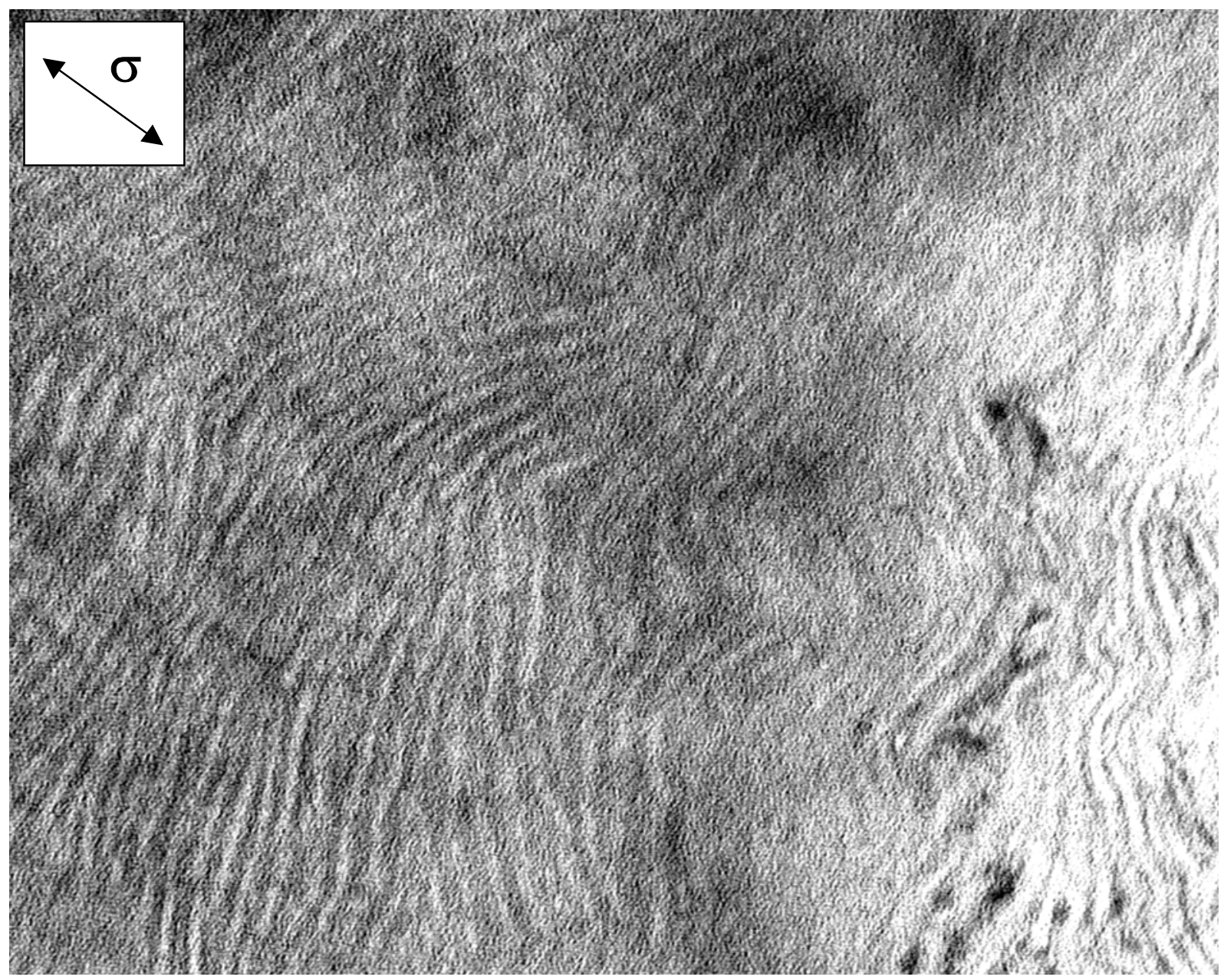

Fig. 11 